\title{
EXTREMAL TRANSITION AND QUANTUM COHOMOLOGY: EXAMPLES OF TORIC DEGENERATION
}

\author{
HIROSHI IRITANI AND JIFU XIAO
}

\begin{abstract}
When a singular projective variety $X_{\text {sing }}$ admits a projective crepant resolution $X_{\text {res }}$ and a smoothing $X_{\mathrm{sm}}$, we say that $X_{\text {res }}$ and $X_{\mathrm{sm}}$ are related by extremal transition. In this paper, we study a relationship between the quantum cohomology of $X_{\mathrm{res}}$ and $X_{\mathrm{sm}}$ in some examples. For three dimensional conifold transition, a result of $\mathrm{Li}$ and Ruan 12 implies that the quantum cohomology of a smoothing $X_{\mathrm{sm}}$ is isomorphic to a certain subquotient of the quantum cohomology of a resolution $X_{\text {res }}$ with the quantum variables of exceptional curves specialized to one. We observe that similar phenomena happen for toric degenerations of $\operatorname{Fl}(1,2,3), \operatorname{Gr}(2,4)$ and $\operatorname{Gr}(2,5)$ by explicit computations.
\end{abstract}

\section{INTRODUCTION}

Let $X_{\text {sing }}$ be a Gorenstein normal projective variety. Suppose that $X_{\text {sing }}$ admits a projective crepant resolution $\pi: X_{\text {res }} \rightarrow X_{\text {sing }}$ and a smoothing $X_{\mathrm{sm}}$ which is projective. The passage from $X_{\text {res }}$ to $X_{\mathrm{sm}}$ is called the extremal transition [14]. When $X_{\text {sing }}$ is a threefold having only ordinary double points as singularities, this is known as conifold transition and has been studied by many people, for example, as a means of constructing new Calabi-Yau threefolds or finding mirrors.

This paper is an attempt to understand the change of quantum cohomology under extremal transition and relate it with the following diagram:

$$
X_{\text {res }} \stackrel{\pi}{\longrightarrow} X_{\text {sing }} \stackrel{r}{\longleftarrow} X_{\text {sm }}
$$

where $\pi$ is a resolution of singularities and $r$ is a (continuous) retraction. Recall that the (small) quantum product $\star$ of a smooth projective variety $X$ defines a commutative ring structure on $Q H^{*}(X)=H^{*}(X) \otimes \mathbb{C} \llbracket q_{1}, \ldots, q_{r} \rrbracket$, where $q_{i}$ 's are the Novikov (quantum) variables associated to a basis of curve classes on $X$ and $r=\operatorname{dim} H^{2}(X)$. This defines the quantum connection (or Dubrovin connection)

$$
\nabla_{q_{i} \frac{\partial}{\partial q_{i}}}=q_{i} \frac{\partial}{\partial q_{i}}+\frac{1}{z}\left(\phi_{i} \star\right) \quad 1 \leq i \leq r
$$

with a parameter $z \in \mathbb{C}^{\times}$, on the trivial bundle over the $q$-space with fiber the cohomology group $H^{*}(X)$. This is flat for all values of $z$. Here $\phi_{1}, \ldots, \phi_{r}$ is a basis of $H^{2}(X)$ dual to the variables $q_{1}, \ldots, q_{r}$.

In the case of threefold conifold transition, Li and Ruan [12] studied the change of Gromov-Witten invariants and functoriality of quantum cohomology. In terms of the quantum connection, their result can be restated as follows:

Theorem 1.1 (see Theorem 3.5 and Corollary 3.6). Let $X_{\mathrm{res}} \rightarrow X_{\mathrm{sing}} \leftarrow X_{\mathrm{sm}}$ be a 3-fold conifold transition. Let $E_{1}, \ldots, E_{k}$ be exceptional curves of $X_{\text {res }}$. 
(a) The quantum connection of $X_{\mathrm{res}}$ is of the form

$$
\nabla^{\mathrm{res}}=\nabla^{\prime}+\sum_{i=1}^{k} N_{i} \frac{d q^{E_{i}}}{1-q^{E_{i}}}
$$

where $\nabla^{\prime}$ is a connection which is regular along $\Delta_{\mathrm{exc}}=\left\{q^{E_{1}}=q^{E_{2}}=\cdots=\right.$ $\left.q^{E_{k}}=1\right\}$ and $N_{i} \in \operatorname{End}\left(H^{*}\left(X_{\text {res }}\right)\right)$ is a nilpotent endomorphism.

(b) The residue endomorphisms $N_{i}$ along $q^{E_{i}}=1$ define the following filtration $0 \subset W \subset V \subset H^{*}\left(X_{\text {res }}\right):$

$$
V:=\bigcap_{i=1}^{k} \operatorname{Ker}\left(N_{i}\right), \quad W:=V \cap V^{\perp}=\bigcap_{i=1}^{k} \operatorname{Ker}\left(N_{i}\right) \cap \sum_{i=1}^{k} \operatorname{Im}\left(N_{i}\right) .
$$

This filtration arises from the diagram (可) as $V=\operatorname{Im} \pi^{*}$ and $W=\pi^{*}\left(\operatorname{Ker} r^{*}\right)$.

(c) The connection $\left.\nabla^{\prime}\right|_{\Delta_{\mathrm{exc}}}$ induces a flat connection on the vector bundle $(V / W) \times$ $\Delta_{\mathrm{exc}} \rightarrow \Delta_{\mathrm{exc}}$ which is isomorphic to the small quantum connection of $X_{\mathrm{sm}}$, under the isomorphism $r^{*} \circ\left(\pi^{*}\right)^{-1}: V / W \cong H^{*}\left(X_{\mathrm{sm}}\right)$.

In particular, the small quantum cohomology $Q H^{*}\left(X_{\mathrm{sm}}\right)$ of $X_{\mathrm{sm}}$ is isomorphic to the subquotient $\left(V / W,\left.\star\right|_{q_{\mathrm{exc}}=1}\right)$ of the quantum cohomology of $X_{\mathrm{res}}$ along the locus where all the exceptional quantum variables $q_{\mathrm{exc}}=\left(q^{E_{1}}, \ldots, q^{E_{k}}\right)$ equal one.

The idea that $Q H^{*}\left(X_{\mathrm{sm}}\right)$ could be described as a subquotient of $Q H^{*}\left(X_{\mathrm{res}}\right)$ with respect to a certan filtration given by monodromy arose out of the discussion of the first author with Tom Coates and Alessio Corti around 2010. We also want to draw attention to a recent paper of Lee-Lin-Wang [11], where they studied the behaviour of $A+B$-theory under conifold transition of Calabi-Yau threefolds.

In this paper we study analogous phenomena for higher dimensional extremal transitions. As studied in 9, 1, a partial flag variety admits a flat degeneration to a singular Gorenstein toric variety $X_{\text {sing, }}$, which in turn admits a toric crepant resolution $X_{\text {res }}$. We study extremal transitions of $\mathrm{Fl}(1,2,3), \operatorname{Gr}(2,4)$ and $\operatorname{Gr}(2,5)$ by explicit computations. A toric degeneration of $\mathrm{Fl}(1,2,3)$ and its resolution is a special case of the threefold conifold transition and we confirm the above result. In the remaining two cases, we find analogous results together with some new phenomena, as follows.

- For $\operatorname{Gr}(2,4)$, the map $r^{*}: H^{*}\left(X_{\text {sing }}\right) \rightarrow H^{*}\left(X_{\mathrm{sm}}\right)$ is not surjective and the subquotient $\left(V / W,\left.\star\right|_{q_{\mathrm{exc}}=1}\right)$ of $H^{*}\left(X_{\mathrm{res}}\right)$ is identified with a proper subring $\operatorname{Im} r^{*} \varsubsetneqq Q H^{*}(\operatorname{Gr}(2,4))$, where $V, W$ are defined by the residue endomorphism $N$ as in (2). If we consider the weight filtration $\left\{W_{\bullet}\right\}$ associated to $N$, we can extend the inclusion $\left(V / W,\left.\star\right|_{q_{\text {exc }}=1}\right) \hookrightarrow Q H^{*}(\operatorname{Gr}(2,4))$ to an isomorphism $W_{0} / W_{-1} \cong Q H^{*}(\operatorname{Gr}(2,4))$. The isomorphism $W_{0} / W_{-1} \cong Q H^{*}(\operatorname{Gr}(2,4))$ however involves an imaginary number.

- For $\operatorname{Gr}(2,5)$, the subquotient $\left(V / W,\left.\star\right|_{q_{\mathrm{exc}}=1}\right)$ is isomorphic to $Q H^{*}(\operatorname{Gr}(2,5))$, where $V, W$ are defined by the residue endomorphisms $N_{2}, N_{3}$ as in (2). In this case, $W \subset \operatorname{Im} \pi^{*} \varsubsetneqq V$ and the isomorphism $V / W \cong H^{*}(\operatorname{Gr}(2,5))$ coincides with $r^{*} \circ\left(\pi^{*}\right)^{-1}$ only on the subspace $\operatorname{Im} \pi^{*} / W$. Also, the quotient $W_{0} / W_{-1}$ associated to the weight filtration $\left\{W_{\bullet}\right\}$ of $a N_{2}+b N_{3}(a \neq 0, b \neq 0)$ has dimension bigger than $\operatorname{dim} H^{*}(\operatorname{Gr}(2,5))$ 
See Theorems 4.1, 5.2, 5.4, 6.1, 6.3 for more details. Note also that $\mathrm{Fl}(1,2,3)$ and $\operatorname{Gr}(2,4)$ are hypersurfaces in toric varieties whereas $\operatorname{Gr}(2,5)$ is not.

This paper is structured as follows. In $\$ 2$, we introduce notation on Gromov-Witten

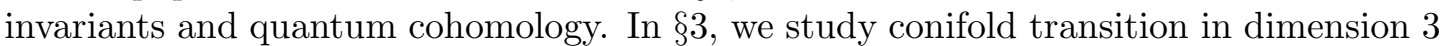
using a result of $\mathrm{Li}$ and Ruan [12]. In $\S$ 近 6 , we study extremal transitions of $\mathrm{Fl}(1,2,3)$, $\operatorname{Gr}(2,4)$ and $\operatorname{Gr}(2,5)$. In $\delta$, we formulate a conjecture for the change of quantum cohomology under extremal transitions of partial flag varieties.

Acknowledgements. H.I. thanks Tom Coates, Alessio Corti, Yunfeng Jiang and Yongbin Ruan for very helpful conversations and related collaborations. H.I. also thanks Yoshinori Namikawa for a very helpful conversation on conifold transition. J.X. thanks Changzheng Li for a helpful conversation on Fano varieties. We thank an anonymous referee who suggested to address the issues dealt in $\$ 6.4$.

\section{Preliminaries}

In this section we fix notation for Gromov-Witten invariants and quantum cohomology. For details on Gromov-Witten theory, we refer the reader to [5] and references therein. In this paper we only consider cohomology classes of even degree and denote by $H^{*}(X)$ the even part $H^{\text {ev }}(X, \mathbb{C})$ of the cohomology group with complex coefficients.

2.1. Gromov-Witten invariants. Let $X$ be a smooth projective variety. For a second homology class $\beta \in H_{2}(X, \mathbb{Z})$ and non-negative integers $g, n$, we denote by $\bar{M}_{g, n}(X, \beta)$ the moduli space of stable maps of degree $\beta$ and genus $g$ with $n$ marked points. This has a virtual fundamental class $\left[\bar{M}_{g, n}(X, \beta)\right]_{\mathrm{vir}} \in H_{2 D}\left(\bar{M}_{g, n}(X, \beta)\right)$ of dimension $D=$ $(1-g)(\operatorname{dim} X-3)+n+\int_{\beta} c_{1}(X)$. Let $\mathrm{ev}_{i}: \bar{M}_{g, n}(X, \beta) \rightarrow X$ be the evaluation map at the $i$ th marked point. Gromov-Witten invariants are defined by

$$
\left\langle\gamma_{1}, \ldots, \gamma_{n}\right\rangle_{g, n, \beta}^{X}=\int_{\left[\bar{M}_{g, n}(X, \beta)\right]_{\mathrm{vir}}} \operatorname{ev}_{1}^{*}\left(\gamma_{1}\right) \cup \cdots \cup \mathrm{ev}_{n}^{*}\left(\gamma_{n}\right)
$$

where $\gamma_{1}, \cdots, \gamma_{n} \in H^{*}(X)$. In this paper, we are mainly interested in three-point genus-zero Gromov-Witten invariants, and the associated small quantum cohomology.

2.2. Quantum cohomology. We choose a basis $\left\{\phi_{0}, \phi_{1}, \ldots, \phi_{N}\right\}$ of $H^{*}(X)$ such that:

(1) $\phi_{0}$ is the identity element of $H^{*}(X)$;

(2) $\phi_{1}, \cdots, \phi_{r}$ form a nef integral basis for $H^{2}(X, \mathbb{Z}) /$ torsion, where $r$ is the rank of $H^{2}(X, \mathbb{Z})$

(3) $\phi_{i}$ is homogeneous.

Let $(\alpha, \beta)=\int_{X} \alpha \cup \beta$ denote the Poincaré pairing. Let $\left\{\phi^{0}, \ldots, \phi^{N}\right\}$ denote the basis dual to $\left\{\phi_{0}, \ldots, \phi_{N}\right\}$ with respect to the Poincaré pairing: $\left(\phi_{i}, \phi^{j}\right)=\delta_{i}^{j}$. Notice that the condition (2) above is equivalent to the condition that the cone spanned by the dual basis $\left\{\phi^{1}, \ldots, \phi^{r}\right\}$ in $H^{2 \operatorname{dim} X-2}(X, \mathbb{R}) \cong H_{2}(X, \mathbb{R})$ contains the cone $\overline{\mathrm{NE}}(X)$ of effective curves (the Mori cone).

Let $q_{1}, \ldots, q_{r}$ be the Novikov variables which are dual to the basis $\left\{\phi_{1}, \ldots, \phi_{r}\right\}$ of $H^{2}(X)$. For $\beta \in H_{2}(X)$, we write

$$
q^{\beta}=q_{1}^{\phi_{1} \cdot \beta} q_{2}^{\phi_{2} \cdot \beta} \cdots q_{r}^{\phi_{r} \cdot \beta} .
$$


Note that if $\beta$ is an effective class, the right-hand side only contains non-negative powers of $q_{1}, \ldots, q_{r}$. We define the Novikov ring to be $\Lambda:=\mathbb{C} \llbracket q_{1}, \ldots, q_{r} \rrbracket$. The small quantum product $\star$ on $H^{*}(X) \otimes \Lambda$ is defined by

$$
(u \star v, w)=\sum_{\beta \in \operatorname{Eff}(X)}\langle u, v, w\rangle_{0,3, \beta}^{X} q^{\beta} .
$$

The product $\star$ defines an associative and commutative ring structure on $H^{*}(X) \otimes \Lambda$. Moreover this is graded with respect to the grading $\operatorname{deg} q_{i}=2 \rho_{i}$ and the usual grading on $H^{*}(X)$, where $c_{1}(X)=\sum_{i=1}^{r} \rho_{i} \phi_{i}$. This is called the small quantum cohomology and denoted by $Q H^{*}(X)$. The structure constants of small quantum cohomology are not known to be convergent in general (as power series in $q_{1}, \ldots, q_{r}$ ); however they are convergent for all the examples in this paper.

2.3. Quantum connection. The quantum cohomology associates a pencil of flat connection, called the quantum connection or Dubrovin connection. This is a flat connection $\nabla$ on the trivial $H^{*}(X)$-bundle over $\mathbb{C}^{r}$ with logarithmic singularities along the normal crossing divisor $q_{1} q_{2} \cdots q_{r}=0$, given by:

$$
\nabla_{q_{i} \frac{\partial}{\partial q_{i}}}=q_{i} \frac{\partial}{\partial q_{i}}+\frac{1}{z}\left(\phi_{i} \star\right)
$$

Here $z \in \mathbb{C}^{\times}$is a parameter of the pencil. The flatness follows from the associativity of the quantum product. When we identify $v=\sum_{i=1}^{r} v^{i} \phi_{i} \in H^{2}(X)$ with the logarithmic vector field $\partial_{v}=\sum_{i=1}^{r} v^{i} q_{i} \frac{\partial}{\partial q_{i}}$ on $\mathbb{C}^{r}$, we can write the quantum connection in the following way:

$$
\nabla_{v}=\partial_{v}+\frac{1}{z}(v \star)
$$

In this paper, we relate the quantum connections of a smoothing and a resolution.

\section{Conifold transition And Quantum COHOMOlOGY}

In this section we describe the change of quantum cohomology under conifold transition in dimension three, using a result of Li-Ruan [12]. Our main result in this section is stated in Theorem 3.5. We observe that the quantum cohomology of a smoothing arises as a limit of the quantum cohomology of a resolution when the quantum variables associated to exceptional curves go to one.

3.1. Geometry of conifold transition. The conifold transition in dimension 3 is a surgery which replaces a $(-1,-1)$-rational curve with a real 3 -sphere. In this section we describe topological properties of the conifold transition. See e.g. [14, 16] for more background material.

Let $X_{\text {sing }}$ be a three-dimensional projective variety whose only singularities are ordinary double points $p_{1}, \ldots, p_{k}$. Recall that an ordinary double point (or $A_{1}$-singularity) is a singularity whose neighbourhood is analytically isomorphic to a neighbourhood of the origin in $\{x y=z w\} \subset \mathbb{C}^{4}$. Let $X_{\text {res }}$ be a small resolution of $X_{\text {sing }}$ and suppose that $X_{\text {sing }}$ admits a smoothing $X_{\mathrm{sm}}$. The passage from $X_{\text {res }}$ to $X_{\mathrm{sm}}$ is called the conifold transition. Since we are interested in Gromov-Witten theory, we assume that both $X_{\text {sing }}$ and $X_{\mathrm{sm}}$ are projective. The inverse image $E_{i}$ of $p_{i}$ in the small resolution $X_{\text {res }}$ is a rational curve whose normal bundle is $\mathcal{O}(-1) \oplus \mathcal{O}(-1)$. The vanishing cycle $S_{i} \subset X_{\text {sm }}$ 
associated to $p_{i}$ is a real 3 -sphere. In topological terms, the conifold transition replaces a neighbourhood $S^{2} \times D^{4}$ of $E_{i}$ with a neighbourhood $D^{3} \times S^{3} \cong T^{*} S_{i}$ of $S_{i}$. There are two natural maps:

- a morphism $\pi: X_{\text {res }} \longrightarrow X_{\text {sing }}$ contracting the rational curves $E_{1}, \ldots, E_{k}$;

- a continuous map $r: X_{\mathrm{sm}} \longrightarrow X_{\text {sing }}$ contracting the real 3 -spheres $S_{1}, \ldots, S_{k}$.

They give the following correspondence between the cohomology groups of the resolution and the smoothing:

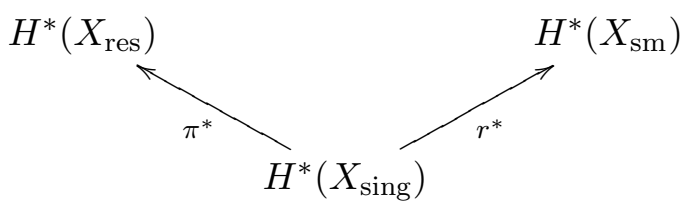

Set $E=E_{1} \cup E_{2} \cup \cdots \cup E_{k} \subset X_{\text {res }}$ and $S=S_{1} \cup S_{2} \cup \cdots \cup S_{k} \subset X_{\text {sm. }}$. The relative cohomology exact sequence gives the following exact sequences:

$$
\begin{aligned}
0 \longrightarrow H^{2}\left(X_{\mathrm{res}}, E\right) & \longrightarrow H^{2}\left(X_{\mathrm{res}}\right) \longrightarrow H^{2}(E) \\
0 \longrightarrow H^{4}\left(X_{\mathrm{res}}, E\right) & \longrightarrow H^{4}\left(X_{\mathrm{res}}\right) \longrightarrow H^{2}\left(X_{\mathrm{sm}}, S\right) \longrightarrow H^{2}\left(X_{\mathrm{sm}}\right) \longrightarrow 0 \\
0 & \longrightarrow H^{4}\left(X_{\mathrm{sm}}, S\right) \longrightarrow H^{4}\left(X_{\mathrm{sm}}\right) \longrightarrow 0
\end{aligned}
$$

Set $P=\left\{p_{1}, \ldots, p_{k}\right\} \subset X_{\text {sing }}$. Then we have $H^{*}\left(X_{\text {res }}, E\right) \cong H^{*}\left(X_{\text {sing }}, P\right) \cong H^{*}\left(X_{\mathrm{sm}}, S\right)$ and $H^{i}\left(X_{\text {sing }}, P\right) \cong H^{i}\left(X_{\text {sing }}\right)$ for $i \geq 2$. Therefore we obtain:

$$
\begin{array}{ll}
0 \rightarrow H^{2}\left(X_{\text {sing }}\right) \stackrel{\pi^{*}}{\rightarrow} H^{2}\left(X_{\text {res }}\right) \rightarrow \mathbb{C}^{k}, & \pi^{*}: H^{4}\left(X_{\text {sing }}\right) \cong H^{4}\left(X_{\text {res }}\right), \\
\mathbb{C}^{k} \rightarrow H^{4}\left(X_{\text {sing }}\right) \stackrel{r^{*}}{\rightarrow} H^{4}\left(X_{\text {sm }}\right) \rightarrow 0, & r^{*}: H^{2}\left(X_{\text {sing }}\right) \cong H^{2}\left(X_{\text {sm }}\right) .
\end{array}
$$

Combining these sequences, we obtain:

$$
\begin{gathered}
0 \longrightarrow H^{2}\left(X_{\mathrm{sm}}\right) \longrightarrow H^{2}\left(X_{\mathrm{res}}\right) \longrightarrow \mathbb{C}^{k}, \\
\left.\mathbb{C}^{k} \longrightarrow H^{4}\left(X_{\mathrm{res}}\right) \longrightarrow X_{\mathrm{sm}}\right) \longrightarrow 0 .
\end{gathered}
$$

Note that the map $H^{2}\left(X_{\text {res }}\right) \rightarrow \mathbb{C}^{k}$ in the first sequence sends a class $\alpha \in H^{2}\left(X_{\text {res }}\right)$ to the vector $\left(\left[E_{1}\right] \cdot \alpha, \ldots,\left[E_{k}\right] \cdot \alpha\right) \in \mathbb{C}^{k}$.

Lemma 3.1. The two sequences in (4) are dual to each other with respect to the Poincaré pairing. In other words, the image of the standard basis vector $e_{i} \in \mathbb{C}^{k}$ in $H^{4}\left(X_{\mathrm{res}}\right)$ under the map $\mathbb{C}^{k} \rightarrow H^{4}\left(X_{\mathrm{res}}\right)$ in the second sequence of (四) is the Poincaré dual of $E_{i}$.

Proof. The dual of the map $\mathbb{C}^{k}=H^{3}(S) \rightarrow H^{4}\left(X_{\mathrm{sm}}, S\right) \cong H^{4}\left(X_{\text {sing }}\right) \cong H^{4}\left(X_{\text {res }}\right)$ is identified with the following boundary map:

$$
H_{4}\left(X_{\text {res }}\right) \cong H_{4}\left(X_{\text {sing }}\right) \cong H_{4}\left(X_{\text {sm }}, S\right) \stackrel{\partial}{\rightarrow} H_{3}(S)=\mathbb{C}^{k}
$$

It suffices to show that this is given by the intersection numbers with the exceptional curves $E_{1}, \ldots, E_{k}$. Take a real 4 -cycle $D \subset X_{\text {res }}$ and suppose that $D$ intersects every $E_{i}$ transversely. Under the conifold transition, each intersection point of $D$ and $E_{i}$ 
is replaced with the 3 -sphere $S_{i}$. Therefore the image of $[D]$ under (5) is given by $\left(E_{i} \cdot D\right)_{i=1}^{k}$. The lemma follows.

Note that the map $\pi^{*}: H^{*}\left(X_{\text {sing }}\right) \rightarrow H^{*}\left(X_{\text {res }}\right)$ is injective and $r^{*}: H^{*}\left(X_{\text {sing }}\right) \rightarrow$ $H^{*}\left(X_{\mathrm{sm}}\right)$ is surjective. The exact sequences (3), (4) and the above lemma imply the following description of $H^{*}\left(X_{\mathrm{sm}}\right)$ as a subquotient of $H^{*}\left(X_{\mathrm{res}}\right)$.

Proposition 3.2. Consider the filtration $0 \subset W \subset V \subset H^{*}\left(X_{\mathrm{res}}\right)$ defined by

$$
W:=\sum_{i=1}^{k} \mathbb{C}\left[E_{i}\right] \subset H^{4}\left(X_{\mathrm{res}}\right), \quad V:=\operatorname{Im} \pi^{*} \cong H^{*}\left(X_{\text {sing }}\right) .
$$

Then we have $V / W \cong H^{*}\left(X_{\mathrm{sm}}\right)$. More precisely, the following holds:

(1) $W$ is the annihilator of $V$ with respect to the Poincaré pairing, i.e. $W=V^{\perp}$. In particular, $V / W$ has a non-degenerate pairing.

(2) The map $r^{*}$ induces an isomorphism $V / W \cong H^{*}\left(X_{\mathrm{sm}}\right)$ which preserves the pairing and the cup product.

Remark 3.3. It is a subtle problem if $X_{\text {sing }}$ admits a smoothing or if the small resolution $X_{\text {res }}$ is projective. In the Calabi-Yau case, there is a criterion due to Friedman, Kawamata and Tian [6, 10, 17] about the smoothability of $X_{\text {sing }}: X_{\text {sing }}$ is smoothable if and only if there exist non-zero rational numbers $\alpha_{1}, \ldots, \alpha_{k} \in \mathbb{Q}^{\times}$such that $\sum_{i=1}^{k} \alpha_{i}\left[E_{i}\right]=0$ in $H_{2}\left(X_{\text {res }}\right)$. In the Fano case, $X_{\text {sing }}$ is always smoothable [6, 15].

3.2. A theorem of $\mathbf{L i}$ and Ruan. We write $\langle\cdots\rangle_{g, n, d}^{\mathrm{res}}$ for Gromov-Witten invariants for $X_{\text {res }}$ and $\langle\cdots\rangle_{g, n, \beta}^{\mathrm{sm}}$ for Gromov-Witten invariants for $X_{\mathrm{sm}}$. Li-Ruan [12] showed the following theorem:

Theorem 3.4 ([12, Theorem B]). Let $v_{1}, \ldots, v_{n}$ be elements of $H^{*}\left(X_{\text {sing }}\right)$ and let $0 \neq$ $\beta \in H_{2}\left(X_{\mathrm{sm}}, \mathbb{Z}\right)$ be a non-zero degree. We have:

$$
\sum_{d: \pi_{*}(d)=\beta}\left\langle\pi^{*}\left(v_{1}\right), \ldots, \pi^{*}\left(v_{n}\right)\right\rangle_{g, n, d}^{\mathrm{res}}=\left\langle r^{*}\left(v_{1}\right), \ldots, r^{*}\left(v_{n}\right)\right\rangle_{g, n, \beta}^{\mathrm{sm}}
$$

The sum in the left-hand side is finite, i.e. $\left\langle\pi^{*}\left(v_{1}\right), \ldots, \pi^{*}\left(v_{n}\right)\right\rangle_{g, n, d}$ with $\pi_{*}(d)=\beta$ with a fixed $\beta$ vanishes except for finitely many $d$.

3.3. Transition of quantum cohomology. We choose a suitable basis of $H_{2}\left(X_{\text {res }}, \mathbb{Z}\right)$. Let $L$ be an ample line bundle over $X_{\text {sing. }}$. Then the line bundle $\pi^{*} L$ is nef on $X_{\text {res }}$, and for any curve $C \subset X_{\text {res }}$, we have $L \cdot C=0$ if and only if $C$ is one of the exceptional curves $E_{1}, \ldots, E_{k}$. Therefore the face $F:=\left\{d \in \overline{\mathrm{NE}}\left(X_{\mathrm{res}}\right): d \cdot \pi^{*} L=0\right\}$ of the Mori cone $\overline{\mathrm{NE}}\left(X_{\text {res }}\right)$ is spanned by the classes of $E_{1}, \ldots, E_{k}$. We choose an integral basis $d_{1}, \ldots, d_{r}$ of $H_{2}\left(X_{\text {res }}, \mathbb{Z}\right) /$ torsion such that

- $d_{1}, \ldots, d_{e}$ span a cone containing the face $F$, where $e=\operatorname{dim} F$;

- $d_{1}, \ldots, d_{r}$ span a cone containing $\overline{\mathrm{NE}}\left(X_{\text {res }}\right)$.

\footnotetext{
${ }^{1}$ We have $e \leq k$. It is possible that $\left[E_{1}\right], \ldots,\left[E_{k}\right]$ are linearly dependent.
} 
Let $q_{1}, \ldots, q_{r}$ be the Novikov variables corresponding to the basis $d_{1}, \ldots, d_{r}$. For any $d=$ $\sum_{i=1}^{r} n_{i} d_{i} \in H_{2}\left(X_{\text {res }}, \mathbb{Z}\right) /$ torsion, we write $q^{d}=q_{1}^{n_{1}} q_{2}^{n_{2}} \cdots q_{r}^{n_{r}}$. By the exact sequence (4), we have

$$
\bigoplus_{i=1}^{k} \mathbb{C}\left[E_{i}\right] \longrightarrow H_{2}\left(X_{\text {res }}\right) \stackrel{\pi_{*}}{\longrightarrow} H_{2}\left(X_{\text {sing }}\right) \cong H_{2}\left(X_{\text {sm }}\right) \longrightarrow 0 .
$$

Therefore $\pi_{*}\left(d_{e+1}\right), \ldots, \pi_{*}\left(d_{r}\right)$ form a basis of $H_{2}\left(X_{\text {sing }}\right) \cong H_{2}\left(X_{\mathrm{sm}}\right)$ and $q_{e+1}, \ldots, q_{r}$ can be identified with Novikov parameters for $X_{\mathrm{sm}}$. Notice that, by Li-Ruan's theorem and by the surjectivity of $r^{*}$, Gromov-Witten invariants of $X_{\mathrm{sm}}$ of degree $\beta \in H_{2}\left(X_{\mathrm{sm}}, \mathbb{Z}\right)$ can be non-zero only when $\beta$ is a linear combination of $\pi_{*}\left(d_{e+1}\right), \ldots, \pi_{*}\left(d_{r}\right)$ with nonnegative coefficients. Therefore the quantum product of $X_{\mathrm{sm}}$ is defined over the ring $\mathbb{C} \llbracket q_{e+1}, \ldots, q_{r} \rrbracket$.

Before stating the result, we explain the meaning of analytic continuation. We will consider analytic continuation of the quantum product of $X_{\text {res }}$ across the locus $\Delta_{\text {exc }}:=$ $\left\{q_{1}=q_{2}=\cdots=q_{e}=1\right\} \subset \mathbb{C}^{r}$ where all the quantum variables associated to exceptional classes equal one. The map $\pi_{*}: H_{2}\left(X_{\text {res }}\right) \rightarrow H_{2}\left(X_{\text {sing }}\right) \cong H_{2}\left(X_{\text {sm }}\right)$ induces a ring homomorphism

$$
\lim _{q_{\mathrm{exc}} \rightarrow 1}:=\lim _{\left(q_{1}, \ldots, q_{e}\right) \rightarrow(1, \ldots, 1)}: \mathbb{C}\left[q_{1}, \ldots, q_{r}\right] \longrightarrow \mathbb{C}\left[q_{e+1}, \ldots, q_{r}\right]
$$

where $q_{\text {exc }}$ stands for quantum variables associated to exceptional curves. However this does not extend to a homomorphism between the Novikov rings $\mathbb{C} \llbracket q_{1}, \ldots, q_{r} \rrbracket$ and $\mathbb{C} \llbracket q_{e+1}, \ldots, q_{r} \rrbracket$. Instead we have a map

$$
\lim _{q_{\mathrm{exc}} \rightarrow 1}: \mathbb{C}\left[q_{1}, \ldots, q_{e}\right] \llbracket q_{e+1}, \ldots, q_{r} \rrbracket \rightarrow \mathbb{C} \llbracket q_{e+1}, \ldots, q_{r} \rrbracket .
$$

Thus, if $v \star w$ is defined over the ring $\mathbb{C}\left[q_{1}, \ldots, q_{e}\right] \llbracket q_{e+1}, \ldots, q_{r} \rrbracket$, we have a well-defined limit $\lim _{q_{\mathrm{exc}} \rightarrow 1} v \star w$.

Theorem 3.5. The quantum cohomology of $X_{\mathrm{sm}}$ is a subquotient of the quantum cohomology of $X_{\mathrm{res}}$ restricted to the locus $\Delta_{\mathrm{exc}}:=\left\{q_{1}=q_{2}=\cdots=q_{e}=1\right\}$ where the Novikov varibles of exceptional curves equal one. More precisely, we have:

(1) The small quantum product of $v \in H^{*}\left(X_{\text {res }}\right)$ is of the form:

$$
(v \star)=\sum_{i=1}^{k}\left(v \cdot\left[E_{i}\right]\right) \frac{q^{E_{i}}}{1-q^{E_{i}}} N_{i}+R(v)
$$

where $R(v) \in \operatorname{End}\left(H^{*}\left(X_{\mathrm{res}}\right)\right) \otimes \mathbb{C}\left[q_{1}, \ldots, q_{e}\right] \llbracket q_{e+1}, \ldots, q_{r} \rrbracket$ is regular along $\Delta_{\text {exc }}$, $\left.R(v)\right|_{q_{e+1}=\cdots=q_{r}=0}$ is the cup product by $v$, and $N_{i} \in \operatorname{End}\left(H^{*}\left(X_{\mathrm{res}}\right)\right)$ is a nilpotent endomorphism defined by $N_{i}(w)=\left(w \cdot\left[E_{i}\right]\right)\left[E_{i}\right]$.

(2) The endomorphisms $N_{i}$ define the filtration $0 \subset W \subset V \subset H^{*}\left(X_{\text {res }}\right)$ by

$$
V:=\bigcap_{i=1}^{k} \operatorname{Ker}\left(N_{i}\right) \quad W:=V \cap \sum_{i=1}^{k} \operatorname{Im}\left(N_{i}\right),
$$

This filtration coincides with the one in Proposition 3.9, i.e. $W=\sum_{i=1}^{k} \mathbb{C}\left[E_{i}\right]$ and $V=\operatorname{Im}\left(\pi^{*}\right) \cong H^{*}\left(X_{\text {sing }}\right)$. 
(3) For $v, w \in V$, the limit $\lim _{q_{\mathrm{exc}} \rightarrow 1} v \star w$ exists and lies in $V \otimes \mathbb{C} \llbracket q_{e+1}, \ldots, q_{r} \rrbracket$. Moreover, the map $r^{*}: V \rightarrow H^{*}\left(X_{\mathrm{sm}}\right)$ satisfies:

$$
r^{*}\left(\lim _{q \mathrm{exc} \rightarrow 1} v \star w\right)=r^{*}(v) \star r^{*}(w) .
$$

Therefore, the isomorphism $V / W \cong H^{*}\left(X_{\mathrm{sm}}\right)$ in Proposition 3.9 intertwines the quantum product of $X_{\text {res }}$ restricted to $\Delta_{\mathrm{exc}}$ with the quantum product of $X_{\mathrm{sm}}$.

In terms of the quantum connection, we can rephrase the above result as follows.

Corollary 3.6. The small quantum connection $\nabla^{\mathrm{res}}$ of $X_{\mathrm{res}}$ is of the form:

$$
\nabla^{\mathrm{res}}=\nabla^{\prime}+\frac{1}{z} \sum_{i=1}^{k} N_{i} \frac{d q^{E_{i}}}{1-q^{E_{i}}}
$$

where $\nabla^{\prime}$ is a connection regular along $\Delta_{\mathrm{exc}}=\left\{q_{1}=\cdots=q_{e}=1\right\}$. The restriction of $\nabla^{\prime}$ to $\Delta_{\text {exc }}$ induces a flat connection on the vector bundle $(V / W) \times \Delta_{\text {exc }} \rightarrow \Delta_{\text {exc }}$ which is isomorphic to the small quantum connection $\nabla^{\mathrm{sm}}$ of $X_{\mathrm{sm}}$ under the natural isomorphism $r^{*}: V / W \cong H^{*}\left(X_{\mathrm{sm}}\right)$.

Remark 3.7. The filtration $0 \subset W \subset V \subset H^{*}\left(X_{\text {res }}\right)$ is the weight filtration associated to the nilpotent endomorphism $\sum_{i=1}^{k} a_{i} N_{i}$ (see e.g. [3, A.2]) for a generic choice of $a_{1}, \ldots, a_{k}$. As we shall see later in $\$[$ for $\operatorname{Gr}(2,5)$, however, the quantum cohomology of a smoothing does not necessarily appear as a subquotient associated with the weight filtration.

Remark 3.8. The monodromy of the quantum connection $\nabla^{\text {res }}$ around the divisor $\left\{q^{E_{i}}=1\right\}$ is conjugate to $\exp \left(2 \pi \sqrt{-1} N_{i} / z\right)$ and is unipotent.

Remark 3.9. The residue of $(v \star)$ along the divisor $q^{E_{i}}=1$ is also computed by LeeLin-Wang [11, Lemma 3.12].

3.4. Proof of Theorem 3.5. We set $V=\operatorname{Im} \pi^{*}$ and $W=\sum_{i=1}^{k} \mathbb{C}\left[E_{i}\right]$ as in Proposition 3.2. Since $V \cong H^{*}\left(X_{\text {sing }}\right)$, we may regard $r^{*}$ as a map from $V$ to $H^{*}\left(X_{\mathrm{sm}}\right)$. Part (2) of Theorem 3.5 follows from part (1) of Theorem 3.5 and the exact sequences (3), (4). Thus it suffices to prove parts (1) and (3) of Theorem 3.5. Part (1) of Theorem 3.5 follows from the following lemma:

Lemma 3.10. Fix $\beta \in H_{2}\left(X_{\mathrm{sm}}, \mathbb{Z}\right) \cong H_{2}\left(X_{\text {sing }}, \mathbb{Z}\right)$ and take $v_{1}, v_{2}, v_{3} \in H^{*}\left(X_{\text {res }}\right)$. Consider the sum

$$
\sum_{d: \pi_{*}(d)=\beta}\left\langle v_{1}, v_{2}, v_{3}\right\rangle_{0,3, d}^{\mathrm{res}} q^{d} .
$$

(1) If $\beta \neq 0$, then the sum is finite;

(2) If $\beta=0$, the sum equals:

$$
\int_{X_{\mathrm{res}}} v_{1} \cup v_{2} \cup v_{3}+\sum_{i=1}^{k}\left(v_{1} \cdot\left[E_{i}\right]\right)\left(v_{2} \cdot\left[E_{i}\right]\right)\left(v_{3} \cdot\left[E_{i}\right]\right) \frac{q^{E_{i}}}{1-q^{E_{i}}} .
$$


Proof. We may assume that $v_{1}, v_{2}, v_{3}$ are homogeneous. Suppose that $\beta \neq 0$. If $v_{1}, v_{2}, v_{3} \in V=\operatorname{Im} \pi^{*}$, the finiteness of the sum (6) follows from Theorem 3.4. If $v_{1} \notin V$, then $v_{1} \in H^{2}\left(X_{\text {res }}\right)$ by homogeneity. Thus we can use the divisor equation to factor out $v_{1}$ :

$$
\text { equation }(6)=\sum_{d: \pi_{*}(d)=\beta}\left(v_{1} \cdot d\right)\left\langle v_{2}, v_{3}\right\rangle_{0,2, d}^{\mathrm{res}} q^{d} .
$$

If in addition $v_{2}, v_{3} \in V$, Theorem 3.4 again shows the finiteness of the sum. The finiteness in the other cases can be similarly shown using the divisor equation and Theorem 3.4.

Next suppose that $\beta=0$. The $d=0$ term in (6) gives $\int_{X_{\text {res }}} v_{1} \cup v_{2} \cup v_{3}$. The only curves in $X_{\text {res }}$ contributing to the sum (6) are multiples of the exceptional curve $E_{i}$. By the dimension axiom, we have $\operatorname{deg} v_{1}+\operatorname{deg} v_{2}+\operatorname{deg} v_{3}=6$. If one of $\operatorname{deg} v_{1}, \operatorname{deg} v_{2}, \operatorname{deg} v_{3}$ is zero, the invariant $\left\langle v_{1}, v_{2}, v_{3}\right\rangle_{0,3, d}$ is zero for $d \neq 0$. Therefore we only need to consider the case where $v_{1}, v_{2}, v_{3} \in H^{2}\left(X_{\text {res }}\right)$. Since the moduli space $M_{0,0}\left(X_{\text {res }}, d\right)$ with $\pi_{*}(d)=0$ consists of multiple covers of some $E_{i}$, we have

$$
\begin{aligned}
\sum_{d \neq 0: \pi_{*}(d)=0}\left\langle v_{1}, v_{2}, v_{3}\right\rangle_{0,3, d}^{\mathrm{res}} & =\sum_{d \neq 0: \pi_{*}(d)=0}\left(v_{1} \cdot d\right)\left(v_{2} \cdot d\right)\left(v_{3} \cdot d\right)\langle\rangle_{0,0, d}^{\mathrm{res}} q^{d} \\
& =\sum_{i=1}^{k} \sum_{n=1}^{\infty}\left(v_{1} \cdot n E_{i}\right)\left(v_{2} \cdot n E_{i}\right)\left(v_{3} \cdot n E_{i}\right) \frac{1}{n^{3}} q^{n E_{i}} \\
& =\sum_{i=1}^{k}\left(v_{1} \cdot E_{i}\right)\left(v_{2} \cdot E_{i}\right)\left(v_{3} \cdot E_{i}\right) \frac{q^{E_{i}}}{1-q^{E_{i}}}
\end{aligned}
$$

by the multiple cover formula 13 for a $(-1,-1)$-curve (each multiple cover of degree $n$ contributes $\left.1 / n^{3}\right)$. The lemma is proved.

Finally we prove part (3) of Theorem 3.5. Suppose that $v, w \in V$. The existence of the limit $\lim _{q_{\mathrm{exc}} \rightarrow 1} v \star w$ follows from Lemma 3.10. We claim that

$$
\lim _{q_{\mathrm{exc}} \rightarrow 1}(u, v \star w)=\left(r^{*}(u), r^{*}(v) \star r^{*}(w)\right)
$$

for all $u \in V$. The left-hand side equals

$$
\sum_{d: \pi_{*}(d)=0}\langle u, v, w\rangle_{0,3, d}^{\mathrm{res}}+\sum_{\beta \neq 0} \sum_{d: \pi_{*}(d)=\beta}\langle u, v, w\rangle_{0,3, d}^{\mathrm{res}} q^{\beta} .
$$

By Lemma 3.10, the first term equals:

$$
\int_{X_{\mathrm{res}}} u \cup v \cup w=\int_{X_{\mathrm{sm}}} r^{*} u \cup r^{*} v \cup r^{*} w
$$

since $u \cdot\left[E_{i}\right]=v \cdot\left[E_{i}\right]=w \cdot\left[E_{i}\right]=0$. We also used the fact that $r^{*}$ preserves the pairing (Proposition 3.2). By Theorem 3.4, the second term of (8) equals:

$$
\sum_{\beta \neq 0}\left\langle r^{*}(u), r^{*}(v), r^{*}(w)\right\rangle_{0,3, \beta}^{\mathrm{sm}} q^{\beta} .
$$

The claim follows. Setting $u=\left[E_{i}\right]$ in equation (7]) and using the fact that $r^{*}\left(\left[E_{i}\right]\right)=0$ from Lemma 3.1, we obtain $\left(\left[E_{i}\right], \lim _{q_{\mathrm{exc}} \rightarrow 1} v \star w\right)=0$. This means that $\lim _{q_{\mathrm{exc}} \rightarrow 1} v \star w$ 
lies in $V$. Using again the fact that $r^{*}$ preserves the pairing, we obtain from equation (17) that

$$
\left(r^{*}(u), r^{*}\left(\lim _{q_{\mathrm{exc}} \rightarrow 1} v \star w\right)\right)=\left(r^{*}(u), r^{*}(v) \star r^{*}(w)\right) .
$$

Since $r^{*}$ is surjective, this shows that $r^{*}\left(\lim _{q_{\mathrm{exc}} \rightarrow 1} v \star w\right)=r^{*}(v) \star r^{*}(w)$. Part (3) of Theorem 3.5 is proved.

\section{Example: $\mathrm{Fl}(1,2,3)$}

In this section we study a conifold transition of $\mathrm{Fl}(1,2,3)$, the space of full flags in $\mathbb{C}^{3}$, confirming the result in the previous section. Consider a toric degeneration of $\operatorname{Fl}(1,2,3)$ given by a family $\left\{F_{t}\right\}_{t \in \mathbb{C}}$ of $(1,1)$-hypersurfaces in $\mathbb{P}^{2} \times \mathbb{P}^{2}$ :

$$
F_{t}=\left\{\left(\left[z_{1}, z_{2}, z_{3}\right],\left[Z_{1}, Z_{2}, Z_{3}\right]\right) \in \mathbb{P}^{2} \times \mathbb{P}^{2} \mid t z_{1} Z_{1}+z_{2} Z_{2}+z_{3} Z_{3}=0\right\} .
$$

Then $F_{t} \cong \operatorname{Fl}(1,2,3)$ for $t \neq 0$ and the central fiber $X_{\text {sing }}:=F_{0}$ is a singular toric variety with an ordinary double point. This admits a small toric crepant resolution $X_{\text {res }} \rightarrow X_{\text {sing. }}$. We study a relationship between the quantum cohomology of $\operatorname{Fl}(1,2,3)$ and $X_{\text {res }}$.

4.1. Quantum cohomology of $\mathrm{Fl}(1,2,3)$. The quantum cohomology ring of a flag variety is well-known see e.g. [8, 4]. Let $L_{1}, L_{2}, L_{3}$ be the line bundles on $\operatorname{Fl}(1,2,3)$ whose fibers at a flag $0 \subset L \subset V \subset \mathbb{C}^{3}$ are given by $L, V / L$ and $\mathbb{C}^{3} / V$ respectively. The cohomology ring of $\mathrm{Fl}(1,2,3)$ is generated by the Chern classes $c_{i}:=-c_{1}\left(L_{i}\right), i=1,2,3$ and:

$$
H^{*}(\mathrm{Fl}(1,2,3)) \cong \mathbb{C}\left[c_{1}, c_{2}, c_{3}\right] /\left\langle\sigma_{1}, \sigma_{2}, \sigma_{3}\right\rangle
$$

where $\sigma_{i}$ is the $i$ th elementary symmetric polynomial of $c_{1}, c_{2}, c_{3}$. A basis of $H^{2}(\mathrm{Fl}(1,2,3))$ is given by

$$
p_{1}:=c_{1}=-c_{1}\left(L_{1}\right), \quad p_{2}:=c_{1}+c_{2}=c_{1}\left(L_{3}\right) .
$$

These classes span the nef cone of $\mathrm{Fl}(1,2,3)$ and satisfy the relations $p_{1}^{2}+p_{2}^{2}-p_{1} p_{2}=0$, $p_{2}^{3}=p_{1}^{3}=0, p_{1}^{2} p_{2}=p_{1} p_{2}^{2}$. The dual basis in $H_{2}(\mathrm{Fl}(1,2,3))$ is:

$$
\beta_{1}=\mathrm{PD}\left(p_{2}^{2}\right), \quad \beta_{2}=\mathrm{PD}\left(p_{1}^{2}\right) .
$$

These classes span the Mori cone: they are represented by fibers of the natural maps $\mathrm{Fl}(1,2,3) \rightarrow \mathrm{Fl}(2,3) \cong\left(\mathbb{P}^{2}\right)^{\star}$ and $\mathrm{Fl}(1,2,3) \rightarrow \mathrm{Fl}(1,3) \cong \mathbb{P}^{2}$ respectively. For an effective class $d=n_{1} \beta_{1}+n_{2} \beta_{2} \in H_{2}(\mathrm{Fl}(1,2,3))$, we write $q^{d}=q_{1}^{n_{1}} q_{2}^{n_{2}}$ with $q_{i}=q^{\beta_{i}}$. Since $c_{1}(\mathrm{Fl}(1,2,3))=2 p_{1}+2 p_{2}$, we have $\operatorname{deg} q_{1}=\operatorname{deg} q_{2}=4$. Consider the basis of $H^{*}(\mathrm{Fl}(1,2,3))$ given by

$$
\left\{1, \quad p_{1}, \quad p_{2}, \quad p_{1}^{2}=\mathrm{PD}\left(\beta_{2}\right), \quad p_{2}^{2}=\mathrm{PD}\left(\beta_{1}\right), \quad p_{1}^{2} p_{2}=\mathrm{PD}([\mathrm{pt}])\right\}
$$


In this basis, the quantum multiplication by $p_{1}$ and $p_{2}$ are given by the following matrices:

$$
p_{1} \star=\left(\begin{array}{cccccc}
0 & q_{1} & 0 & 0 & 0 & q_{1} q_{2} \\
1 & 0 & 0 & 0 & 0 & 0 \\
0 & 0 & 0 & q_{1} & 0 & 0 \\
0 & 1 & 1 & 0 & 0 & 0 \\
0 & 0 & 1 & 0 & 0 & q_{1} \\
0 & 0 & 0 & 0 & 1 & 0
\end{array}\right) \quad p_{2} \star=\left(\begin{array}{cccccc}
0 & 0 & q_{2} & 0 & 0 & q_{1} q_{2} \\
0 & 0 & 0 & 0 & q_{2} & 0 \\
1 & 0 & 0 & 0 & 0 & 0 \\
0 & 1 & 0 & 0 & 0 & q_{2} \\
0 & 1 & 1 & 0 & 0 & 0 \\
0 & 0 & 0 & 1 & 0 & 0
\end{array}\right)
$$

4.2. Quantum cohomology of $X_{\text {res }}$. The singular fiber $X_{\text {sing }}=F_{0}$ is a toric variety and the corresponding fan is given by the following data: one-dimensional cones are spanned by:

$$
\begin{array}{ll}
r_{1}=(0,0,1), \quad r_{2}=(1,1,-1), & r_{3}=(0,1,0), \\
r_{4}=(0,-1,0), \quad r_{5}=(1,0,0), & r_{6}=(-1,0,0),
\end{array}
$$

and the full-dimensional cones are given by:

$$
\begin{array}{cccc}
\left\langle r_{1}, r_{3}, r_{6}\right\rangle, & \left\langle r_{1}, r_{4}, r_{5}\right\rangle, & \left\langle r_{1}, r_{4}, r_{6}\right\rangle, & \left\langle r_{1}, r_{2}, r_{3}, r_{5}\right\rangle, \\
\left\langle r_{2}, r_{4}, r_{5}\right\rangle, & \left\langle r_{2}, r_{4}, r_{6}\right\rangle, & \left\langle r_{2}, r_{3}, r_{6}\right\rangle .
\end{array}
$$

A small resolution $X_{\text {res }}$ of $X_{\text {sing }}$ is given by dividing the cone $\left\langle r_{1}, r_{2}, r_{3}, r_{5}\right\rangle$ into the two simplicial cones $\left\langle r_{1}, r_{3}, r_{5}\right\rangle$ and $\left\langle r_{2}, r_{3}, r_{5}\right\rangle$. Let $R_{1}, \ldots, R_{6} \in H^{2}\left(X_{\text {res }}\right)$ be the classes of the prime toric divisors corresponding to the one-dimensional cones $\left\langle r_{1}\right\rangle, \ldots,\left\langle r_{6}\right\rangle$. The cohomology ring of $X_{\text {res }}$ is generated by $R_{1}, \ldots, R_{6}$ with the relations $R_{1}=R_{2}$, $R_{2}+R_{3}=R_{4}, R_{2}+R_{5}=R_{6}, R_{1} R_{2}=R_{3} R_{4}=R_{5} R_{6}=0$. We choose a basis $\left\{\mathfrak{p}_{1}, \mathfrak{p}_{2}, \mathfrak{p}_{3}\right\}$ of $H^{2}\left(X_{\text {res }}\right)$ as

$$
\mathfrak{p}_{1}:=R_{4}, \quad \mathfrak{p}_{2}:=R_{6}, \quad \mathfrak{p}_{3}:=R_{2} .
$$

They span the nef cone of $X_{\text {res }}$ and satisfy the relations $\mathfrak{p}_{1}\left(\mathfrak{p}_{1}-\mathfrak{p}_{3}\right)=\mathfrak{p}_{2}\left(\mathfrak{p}_{2}-\mathfrak{p}_{3}\right)=$ $\mathfrak{p}_{3}^{2}=0$. The dual basis in $H_{2}\left(X_{\text {res }}\right)$ is given by:

$$
\beta_{1}:=\operatorname{PD}\left(\mathfrak{p}_{2} \mathfrak{p}_{3}\right), \quad \beta_{2}:=\operatorname{PD}\left(\mathfrak{p}_{1} \mathfrak{p}_{3}\right), \quad \beta_{3}:=\operatorname{PD}\left(R_{3} R_{5}\right)=\operatorname{PD}\left(\mathfrak{p}_{1} \mathfrak{p}_{2}-\mathfrak{p}_{1} \mathfrak{p}_{3}-\mathfrak{p}_{2} \mathfrak{p}_{3}\right)
$$

They span the Mori cone of $X_{\text {res }}$. The class $\beta_{3}$ is represented by the exceptional curve in $X_{\text {res }}$.

We can compute the quantum product of $X_{\text {res }}$ by using Givental's mirror theorem [7]. The computation will be illustrated in Appendix A for the example in 86 . For $d=n_{1} \beta_{1}+n_{2} \beta_{2}+n_{3} \beta_{3} \in H_{2}\left(X_{\text {res }}\right)$, we write $q^{d}=q_{1}^{n_{1}} q_{2}^{n_{2}} q_{3}^{n_{3}}$, setting $q_{i}=q^{\beta_{i}}$. Since $c_{1}\left(X_{\text {res }}\right)=2 \mathfrak{p}_{1}+2 \mathfrak{p}_{2}$, we have $\operatorname{deg} q_{1}=\operatorname{deg} q_{2}=4$ and $\operatorname{deg} q_{3}=0$. Consider the following basis of $H^{*}\left(X_{\text {res }}\right)$ :

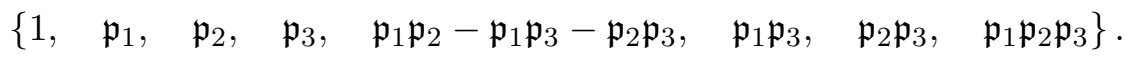


In this basis, the quantum product by $\mathfrak{p}_{1}, \mathfrak{p}_{2}, \mathfrak{p}_{3}$ are represented by the following matrices:

$$
\begin{aligned}
\mathfrak{p}_{1} \star & =\left(\begin{array}{cccccccc}
0 & q_{1} & 0 & 0 & 0 & 0 & 0 & q_{1} q_{2} q_{3} \\
1 & 0 & 0 & 0 & 0 & 0 & 0 & 0 \\
0 & 0 & 0 & 0 & q_{1}\left(1-q_{3}\right) & q_{1} q_{3} & 0 & 0 \\
0 & 0 & 0 & 0 & -q_{1}\left(1-q_{3}\right) & q_{1}\left(1-q_{3}\right) & 0 & 0 \\
0 & 0 & 1 & 0 & 0 & 0 & 0 & 0 \\
0 & 1 & 1 & 1 & 0 & 0 & 0 & 0 \\
0 & 0 & 1 & 0 & 0 & 0 & 0 & q_{1} \\
0 & 0 & 0 & 0 & 0 & 0 & 1 & 0
\end{array}\right) \\
\mathfrak{p}_{2} \star & =\left(\begin{array}{cccccccc}
0 & 0 & q_{2} & 0 & 0 & 0 & 0 & q_{1} q_{2} q_{3} \\
0 & 0 & 0 & 0 & q_{2}\left(1-q_{3}\right) & 0 & q_{2} q_{3} & 0 \\
1 & 0 & 0 & 0 & 0 & 0 & 0 & 0 \\
0 & 0 & 0 & 0 & -q_{2}\left(1-q_{3}\right) & 0 & q_{2}\left(1-q_{3}\right) & 0 \\
0 & 1 & 0 & 0 & 0 & 0 & 0 & 0 \\
0 & 1 & 0 & 0 & 0 & 0 & 0 & q_{2} \\
0 & 1 & 1 & 1 & 0 & 0 & 0 & 0 \\
0 & 0 & 0 & 0 & 0 & 1 & 0 & 0
\end{array}\right) \\
\mathfrak{p}_{3} \star & =\left(\begin{array}{cccccccc}
0 & 0 & 0 & 0 & 0 & 0 & 0 & q_{1} q_{2} q_{3} \\
0 & 0 & 0 & 0 & -q_{2} q_{3} & 0 & q_{2} q_{3} & 0 \\
0 & 0 & 0 & 0 & -q_{1} q_{3} & q_{1} q_{3} & 0 & 0 \\
1 & 0 & 0 & 0 & q_{3}\left(q_{1}+q_{2}\right) & -q_{1} q_{3} & -q_{2} q_{3} & 0 \\
0 & 0 & 0 & \frac{q_{3}}{1-q_{3}} & 0 & 0 & 0 & 0 \\
0 & 1 & 0 & 0 & 0 & 0 & 0 & 0 \\
0 & 0 & 1 & 0 & 0 & 0 & 0 & 0 \\
0 & 0 & 0 & 0 & 1 & 0 & 0 & 0
\end{array}\right)
\end{aligned}
$$

4.3. Comparison of quantum cohomology. We write $X_{\mathrm{sm}}$ for $\mathrm{Fl}(1,2,3)$. Recall from $\$ 3.1$ that we have natural maps

$$
X_{\text {res }} \stackrel{\pi}{\rightarrow} X_{\text {sing }} \stackrel{r}{\leftarrow} X_{\text {sm }} .
$$

The map $\pi^{*}: H^{*}\left(X_{\text {sing }}\right) \rightarrow H^{*}\left(X_{\text {res }}\right)$ is injective and has the image:

$$
\pi^{*}\left(H^{*}\left(X_{\text {sing }}\right)\right)=\left\langle 1, \mathfrak{p}_{1}, \mathfrak{p}_{2}, \mathfrak{p}_{1} \mathfrak{p}_{2}-\mathfrak{p}_{1} \mathfrak{p}_{3}-\mathfrak{p}_{2} \mathfrak{p}_{3}, \mathfrak{p}_{1} \mathfrak{p}_{3}, \mathfrak{p}_{2} \mathfrak{p}_{3}, \mathfrak{p}_{1} \mathfrak{p}_{2} \mathfrak{p}_{2}\right\rangle .
$$

The map $r^{*}: H^{*}\left(X_{\text {sing }}\right) \rightarrow H^{*}\left(X_{\mathrm{sm}}\right)$ is sujective with kernel:

$$
\pi^{*}\left(\operatorname{Ker}\left(r^{*}\right)\right)=\left\langle\mathfrak{p}_{1} \mathfrak{p}_{2}-\mathfrak{p}_{1} \mathfrak{p}_{3}-\mathfrak{p}_{2} \mathfrak{p}_{3}\right\rangle=\left\langle\operatorname{PD}\left(\beta_{3}\right)\right\rangle .
$$

On the second homology groups, the maps $\pi, r$ induce a map?

$$
\left(r_{*}\right)^{-1} \pi_{*}: H_{2}\left(X_{\mathrm{res}}\right) \rightarrow H_{2}\left(X_{\mathrm{sm}}\right), \quad \beta_{1} \mapsto \beta_{1}, \beta_{2} \mapsto \beta_{2}, \beta_{3} \mapsto 0 .
$$

\footnotetext{
${ }^{2}$ Note that $r_{*}$ on $H_{2}$ is an isomorphism.
} 
This gives rise to the map $\lim _{q_{3} \rightarrow 1}: \mathbb{C}\left[q_{1}, q_{2}, q_{3}\right] \rightarrow \mathbb{C}\left[q_{1}, q_{2}\right]$ between Novikov rings. The residue of the quantum multiplication by $\mathfrak{p}_{3}$ on $H^{*}\left(X_{\text {res }}\right)$ along $q_{3}=1$ is:

$$
N=\operatorname{Res}_{q_{3}=1}\left(\mathfrak{p}_{3} \star\right)=\left(\begin{array}{cccccccc}
0 & 0 & 0 & 0 & 0 & 0 & 0 & 0 \\
0 & 0 & 0 & 0 & 0 & 0 & 0 & 0 \\
0 & 0 & 0 & 0 & 0 & 0 & 0 & 0 \\
0 & 0 & 0 & 0 & 0 & 0 & 0 & 0 \\
0 & 0 & 0 & -1 & 0 & 0 & 0 & 0 \\
0 & 0 & 0 & 0 & 0 & 0 & 0 & 0 \\
0 & 0 & 0 & 0 & 0 & 0 & 0 & 0 \\
0 & 0 & 0 & 0 & 0 & 0 & 0 & 0
\end{array}\right)
$$

It is nilpotent and induces the weight filtration on $H^{*}\left(X_{\text {res }}\right)$ :

$$
0 \subset \operatorname{Im} N \subset \operatorname{Ker} N \subset H^{*}\left(X_{\text {res }}\right) \text {. }
$$

The computation in $\S 4.1$, 4.2 shows the following proposition, which confirms the general argument in $\oint$ 3.

Theorem 4.1. The weight filtration (9) defined by the nilpotent operator $N=$ $\operatorname{Res}_{q_{3}=1}\left(\mathfrak{p}_{3} \star\right)$ coincides with the filtration

$$
0 \subset \pi^{*}\left(\operatorname{Ker} r^{*}\right) \subset \operatorname{Im} \pi^{*} \subset H^{*}\left(X_{\mathrm{res}}\right) .
$$

The quantum multiplication by $\mathfrak{p}_{1}, \mathfrak{p}_{2}$ on $H^{*}\left(X_{\mathrm{res}}\right)$ are regular at $q_{3}=1$ and the operators induced by $\lim _{q_{3} \rightarrow 1} \mathfrak{p}_{1} \star, \lim _{q_{3} \rightarrow 1} \mathfrak{p}_{2} \star$ on

$$
\operatorname{Ker} N / \operatorname{Im} N \cong H^{*}(\operatorname{Fl}(1,2,3))
$$

coincide with the quantum multiplication by $p_{1}, p_{2}$ on $H^{*}(\mathrm{Fl}(1,2,3))$. Here note that $\mathfrak{p}_{i} \in \operatorname{Im} \pi^{*}$ and $p_{i}=r^{*}\left(\pi^{*}\right)^{-1} \mathfrak{p}_{i}$ for $i=1,2$.

\section{EXAMPLE: $\operatorname{Gr}(2,4)$}

In this section we study an extremal transition of $\operatorname{Gr}(2,4)$, the space of complex two planes in $\mathbb{C}^{4}$. By the Plücker embedding, $\operatorname{Gr}(2,4)$ can be realized as a quadric in $\mathbb{P}^{5}=\mathbb{P}\left(\wedge^{2} \mathbb{C}^{4}\right)$. Consider a toric degeneration of $\operatorname{Gr}(2,4)$ given by a family $\left\{F_{t}\right\}_{t \in \mathbb{C}}$ of quadric hyperplanes in $\mathbb{P}^{5}$ :

$$
F_{t}=\left\{\left[Z_{12}, Z_{13}, Z_{14}, Z_{23}, Z_{24}, Z_{34}\right] \in \mathbb{P}^{5} \mid Z_{12} Z_{34}-Z_{13} Z_{24}+t Z_{14} Z_{23}=0\right\} .
$$

Then $F_{t} \cong \operatorname{Gr}(2,4)$ for $t \neq 0$ and the central fiber $X_{\text {sing }}:=F_{0}$ is a singular toric variety with a transversal $A_{1}$-singularity along $\left(Z_{12}=Z_{34}=Z_{13}=Z_{24}=0\right) \cong \mathbb{P}^{1}$. This singular variety admits a small toric crepant resolution $X_{\text {res }} \rightarrow X_{\text {sing. }}$. We study a relationship between the quantum cohomology of $\operatorname{Gr}(2,4)$ and $X_{\text {res. }}$.

5.1. Quantum cohomology of $\operatorname{Gr}(2,4)$. Let $T^{\star}$ be the dual tautological bundle of $\operatorname{Gr}(2,4)$. The cohomology ring of $\operatorname{Gr}(2,4)$ is generated by the Chern classes $c_{1}\left(T^{\star}\right)$ and $c_{2}\left(T^{\star}\right)$. Fix a complete flag $0 \subset E_{1} \subset E_{2} \subset E_{3} \subset E_{4}=\mathbb{C}^{4}$ in $\mathbb{C}^{4}$. Consider the following cycles:

$$
\begin{aligned}
& D=\left\{V \in \operatorname{Gr}(2,4): \operatorname{dim}\left(V \cap E_{2}\right)=1\right\} \\
& \Delta=\left\{V \in \operatorname{Gr}(2,4): V \subset E_{3}\right\} \\
& C=\left\{V \in \operatorname{Gr}(2,4): E_{1} \subset V \subset E_{3}\right\}
\end{aligned}
$$


Their Poincaré duals are denoted respectively by $d, \delta, c$. We know that $d=c_{1}\left(T^{\star}\right)$ and $\delta=c_{2}\left(T^{\star}\right)$ and $c=d \delta=d^{3} / 2$. The cohomology ring is given by

$$
H^{*}(\operatorname{Gr}(2,4)) \cong \mathbb{C}[d, \delta] /\left\langle d^{3}-2 d \delta, d^{2} \delta-\delta^{2}\right\rangle
$$

We choose an additive basis of $H^{*}(\operatorname{Gr}(2,4))$ as follows:

$$
1, \quad d, \quad d^{2}, \quad d^{2}-2 \delta, \quad d^{3}, \quad d^{4}
$$

Let $q$ be the Novikov variable dual to $d \in H^{2}(\operatorname{Gr}(2,4))$. We have $\operatorname{deg} q=8$. We use the quantum Schubert calculus [2, [] to compute the quantum product of $d$. Under the above basis, the quantum product matrix of $d$ is:

$$
d \star=\left(\begin{array}{cccccc}
0 & 0 & 0 & 0 & 2 q & 0 \\
1 & 0 & 0 & 0 & 0 & 2 q \\
0 & 1 & 0 & 0 & 0 & 0 \\
0 & 0 & 0 & 0 & 0 & 0 \\
0 & 0 & 1 & 0 & 0 & 0 \\
0 & 0 & 0 & 0 & 1 & 0
\end{array}\right)
$$

and the quantum product matrix of $\delta$ is:

$$
\delta \star=\left(\begin{array}{cccccc}
0 & 0 & q & q & 0 & 0 \\
0 & 0 & 0 & 0 & 2 q & 0 \\
\frac{1}{2} & 1 & 0 & 0 & 0 & 2 q \\
-\frac{1}{2} & 0 & 0 & 0 & 0 & 0 \\
0 & \frac{1}{2} & 0 & 0 & 0 & 0 \\
0 & 0 & \frac{1}{2} & -\frac{1}{2} & 0 & 0
\end{array}\right) .
$$

5.2. Quantum cohomology of $X_{\text {res }}$. The fan for the singular toric variety $X_{\text {sing }}$ is as follows: it is a 4-dimensional fan whose 1-dimensional cones are spanned by

$$
\begin{aligned}
& r_{1}=(1,0,0,0), \quad r_{2}=(-1,0,1,0), \quad r_{3}=(0,0,-1,1), \\
& r_{4}=(-1,1,0,0), \quad r_{5}=(0,-1,0,1), \quad r_{6}=(0,0,0,-1) .
\end{aligned}
$$

This is a complete fan whose top dimensional cones are:

$$
\begin{array}{lll}
\left\langle r_{1}, r_{3}, r_{5}, r_{6}\right\rangle, & \left\langle r_{1}, r_{2}, r_{4}, r_{6}\right\rangle, & \left\langle r_{2}, r_{3}, r_{4}, r_{5}, r_{6}\right\rangle, \\
\left\langle r_{1}, r_{2}, r_{5}, r_{6}\right\rangle, & \left\langle r_{1}, r_{3}, r_{4}, r_{6}\right\rangle, & \left\langle r_{1}, r_{2}, r_{3}, r_{4}, r_{5}\right\rangle .
\end{array}
$$

Note that there are two non-simplicial 4-dimensional cones. We divide these cones as follows:

- divide $\left\langle r_{2}, r_{3}, r_{4}, r_{5}, r_{6}\right\rangle$ into $\left\langle r_{2}, r_{3}, r_{4}, r_{6}\right\rangle$ and $\left\langle r_{2}, r_{3}, r_{5}, r_{6}\right\rangle$.

- divide $\left\langle r_{1}, r_{2}, r_{3}, r_{4}, r_{5}\right\rangle$ into $\left\langle r_{1}, r_{2}, r_{3}, r_{4}\right\rangle$ and $\left\langle r_{1}, r_{2}, r_{3}, r_{5}\right\rangle$.

Then we get a smooth fan. This fan corresponds to a smooth toric variety which we denote by $X_{\text {res }}$. Let $R_{i}$ denote the class of the toric divisor corresponding to the ray $\left\langle r_{i}\right\rangle$. There are linear relations: $R_{1}=R_{2}+R_{4}, R_{4}=R_{5}, R_{2}=R_{3}, R_{3}+R_{5}=R_{6}$. The cohomology ring of $X_{\text {res }}$ is given by:

$$
H^{*}\left(X_{\mathrm{sm}}\right)=\mathbb{C}\left[R_{1}, R_{4}\right] /\left\langle R_{4}^{2}, R_{1}^{4}-2 R_{1}^{3} R_{4}\right\rangle .
$$

We choose a basis $\left\{m_{1}, m_{2}\right\}$ of $H^{2}\left(X_{\text {res }}\right)$ as $m_{1}=R_{1}, m_{2}=R_{4}$. They span the nef cone of $X_{\text {res. }}$. The dual basis in $H_{2}\left(X_{\text {res }}\right)$ is given by $\beta_{1}=\operatorname{PD}\left(R_{1} R_{2} R_{4}\right)$ and $\beta_{2}=$ 
$\mathrm{PD}\left(R_{1} R_{2} R_{3}\right)$. They span the Mori cone of $X_{\text {res }}$. The class $\beta_{2}$ is represented by an exceptional curve.

We compute the quantum product of $X_{\text {res }}$ using Givental's mirror theorem [7], see Appendix A for the method. For $d=n_{1} \beta_{1}+n_{2} \beta_{2} \in H_{2}\left(X_{\text {res }}\right)$, we write $q^{d}=q_{1}^{n_{1}} q_{2}^{n_{2}}$, where $q_{i}=q^{\beta_{i}}$. We have $\operatorname{deg} q_{1}=8$ and $\operatorname{deg} q_{2}=0$. We choose the following bases for the cohomology ring of $X_{\text {res }}$ :

$$
\left\{1, m_{1}, m_{1}-2 m_{2}, m_{1}^{2}, m_{1}^{2}-2 m_{1} m_{2}, m_{1}^{3}, m_{1}^{3}-2 m_{1}^{2} m_{2}, m_{1}^{4}=2[\mathrm{pt}]\right\} .
$$

Under this basis, the quantum product matrices of the divisors $m_{1}$ and $m_{2}$ are as follows:

$$
\begin{aligned}
& m_{1} \star=\left(\begin{array}{cccccccc}
0 & 0 & 0 & 0 & 0 & q_{1}\left(1+q_{2}\right) & q_{1}\left(1-q_{2}\right) & 0 \\
1 & 0 & 0 & 0 & 0 & 0 & 0 & q_{1}\left(1+q_{2}\right) \\
0 & 0 & 0 & 0 & 0 & 0 & 0 & -q_{1}\left(1-q_{2}\right) \\
0 & 1 & 0 & 0 & 0 & 0 & 0 & 0 \\
0 & 0 & 1 & 0 & 0 & 0 & 0 & 0 \\
0 & 0 & 0 & 1 & 0 & 0 & 0 & 0 \\
0 & 0 & 0 & 0 & 1 & 0 & 0 & 0 \\
0 & 0 & 0 & 0 & 0 & 1 & 0 & 0
\end{array}\right) \\
& m_{2} \star=\left(\begin{array}{cccccccc}
0 & 0 & 0 & 0 & 0 & q_{1}\left(1-q_{2}\right) & 2 q_{1} q_{2} & 0 \\
0 & 0 & 0 & 0 & 0 & 0 & 0 & q_{1}\left(1-q_{2}\right) \\
1 & 0 & 2 & 0 & 0 & 0 & 0 & -q_{1}\left(1+q_{2}\right) \\
0 & 0 & -1 & 0 & 0 & 0 & 0 & 0 \\
0 & 1 & \frac{2\left(1+q_{2}\right)}{1-q_{2}} & 0 & 0 & 0 & 0 & 0 \\
0 & 0 & 0 & 0 & -1 & 0 & 0 & 0 \\
0 & 0 & 0 & 1 & \frac{2\left(1+q_{2}\right)}{1-q_{2}} & 0 & 0 & 0 \\
0 & 0 & 0 & 0 & 0 & 0 & 0 & 0
\end{array}\right)
\end{aligned}
$$

5.3. Comparison of quantum cohomology. The residue of the quantum product matrix of $m_{2}$ at $q_{2}=1$ is

$$
N=\operatorname{Res}_{q_{2}=1}\left(m_{2} \star\right)=\left(\begin{array}{cccccccc}
0 & 0 & 0 & 0 & 0 & 0 & 0 & 0 \\
0 & 0 & 0 & 0 & 0 & 0 & 0 & 0 \\
0 & 0 & 0 & 0 & 0 & 0 & 0 & 0 \\
0 & 0 & 0 & 0 & 0 & 0 & 0 & 0 \\
0 & 0 & 4 & 0 & 0 & 0 & 0 & 0 \\
0 & 0 & 0 & 0 & 0 & 0 & 0 & 0 \\
0 & 0 & 0 & 0 & 4 & 0 & 0 & 0 \\
0 & 0 & 0 & 0 & 0 & 0 & 0 & 0
\end{array}\right) .
$$

The residue $N$ defines the filtration $0 \subset W \subset V \subset H^{*}\left(X_{\text {res }}\right)$ as:

$$
\begin{aligned}
V & :=\operatorname{Ker} N=\operatorname{Span}\left\{1, m_{1}, m_{1}^{2}, m_{1}^{3}, m_{1}^{4}, m_{1}^{3}-2 m_{1}^{2} m_{2}\right\} \\
W & :=\operatorname{Ker} N \cap \operatorname{Im} N=\mathbb{C}\left(m_{1}^{3}-2 m_{1}^{2} m_{2}\right) .
\end{aligned}
$$

This filtration arises from the correspondence $X_{\text {res }} \rightarrow X_{\text {sing }} \leftarrow X_{\mathrm{sm}}:=\operatorname{Gr}(2,4)$ as follows:

Proposition 5.1. Let $\pi: X_{\text {res }} \rightarrow X_{\text {sing }}$ and $r: X_{\mathrm{sm}}=\operatorname{Gr}(2,4) \rightarrow X_{\text {sing }}$ be natural maps associated to the resolution and the smoothing. 
(1) The singular cohomology group of $X_{\text {sing }}$ is given by the table:

\begin{tabular}{l|lllllllll} 
degree $p$ & 0 & 1 & 2 & 3 & 4 & 5 & 6 & 7 & 8 \\
\hline$H^{p}\left(X_{\text {sing }}\right)$ & $\mathbb{C}$ & 0 & $\mathbb{C}$ & 0 & $\mathbb{C}$ & 0 & $\mathbb{C}^{2}$ & 0 & $\mathbb{C}$
\end{tabular}

(2) The map $\pi^{*}: H^{*}\left(X_{\text {sing }}\right) \rightarrow H^{*}\left(X_{\text {res }}\right)$ is injective and $\operatorname{Im} \pi^{*}=V$.

(3) The map $r^{*}: H^{*}\left(X_{\text {sing }}\right) \rightarrow H^{*}\left(X_{\mathrm{sm}}\right)$ is neither surjective nor injective; we have $\pi^{*}\left(\operatorname{Ker} r^{*}\right)=W$ and $\operatorname{Im} r^{*}=\operatorname{Span}\left\{1, d, d^{2}, d^{3}, d^{4}\right\}$.

(4) The map $r^{*} \circ\left(\pi^{*}\right)^{-1}: V \rightarrow H^{*}\left(X_{\mathrm{sm}}\right)$ sends $m_{1}^{i}$ to $d^{i}$ for $0 \leq i \leq 4$ and $m_{1}^{3}-$ $2 m_{1}^{2} m_{2}$ to zero.

Proof. Note that the non-singular locus $Y$ of $X_{\text {sing }}$ is isomorphic to the total space of $\mathcal{O}(1,1)^{\oplus 2}$ over $\mathbb{P}^{1} \times \mathbb{P}^{1}$. We consider the Mayer-Vietoris exact sequence associated to $Y$ and a neighbourhood $\nu$ of the singular locus $\mathbb{P}^{1}$. The intersection $\nu \cap Y$ is homotopic to the 3 -sphere bundle associated to $\mathcal{O}(1,1)^{\oplus 2} \rightarrow \mathbb{P}^{1} \times \mathbb{P}^{1}$ and the cohomology of $\nu \cap Y$ can be easily computed by the Gysin sequence: we have

$$
H^{*}(N \cap Y)=\mathbb{C}, 0, \mathbb{C}^{2}, 0,0, \mathbb{C}^{2}, 0, \mathbb{C} \text { for } *=0,1,2,3,4,5,6,7 .
$$

Then the Mayer-Vietoris sequence gives the result for $H^{*}\left(X_{\text {sing }}\right)$. To prove the statement about $\pi^{*}$, we consider the hypercohomology spectral sequence for $\mathbb{H}^{*}\left(X_{\text {sing }}, \mathbb{R} \pi_{*} \mathbb{C}\right)=$ $H^{*}\left(X_{\text {res }}\right)$. Since we have

$$
R^{j} \pi_{* \underline{\mathbb{C}}}= \begin{cases}\underline{\mathbb{C}} & j=0 \\ \iota_{*} \mathbb{C}_{\mathbb{P}^{1}} & j=2 \\ 0 & \text { otherwise }\end{cases}
$$

where $\iota: \mathbb{P}^{1} \rightarrow X_{\text {sing }}$ is the inclusion of the singular locus, the spectral sequence degenerates at the $E_{2}$ term $H^{j}\left(R^{i} \pi_{*} \underline{\mathbb{C}}\right)$; this shows that $\pi^{*}$ is injective. Since the image of $\pi^{*}$ contains the pull-back $m_{1}$ of the ample class $\alpha:=c_{1}(\mathcal{O}(1))$ on $X_{\text {sing }}$, it follows that $\operatorname{Im} \pi^{*}=V$. On the other hand, $r^{*}$ also sends the ample class $\alpha$ to $d=c_{1}(\mathcal{O}(1)) \in H^{2}\left(X_{\mathrm{sm}}\right)$ and it follows that $\operatorname{Im} r^{*}=\operatorname{Span}\left\{1, d, d^{2}, d^{3}, d^{4}\right\}$. Let $x \in H^{6}\left(X_{\text {sing }}\right)$ be a generator of the kernel of $r^{*}$. Then we have $\alpha \cup x=0$ in $H^{8}\left(X_{\text {sing }}\right)$ (as otherwise we have $\left.0 \neq r^{*}(\alpha \cup x)=r^{*}(\alpha) \cup r^{*}(x)=0\right)$. Therefore $0=\pi^{*}(\alpha \cup x)=m_{1} \cup \pi^{*}(x)$. This shows that $\pi^{*}(x)$ is a multiple of $m_{1}^{3}-2 m_{1}^{2} m_{2}$.

The computation in $\$ 5.15 .2$ implies the following theorem:

Theorem 5.2. The filtration $0 \subset W \subset V \subset H^{*}\left(X_{\text {res }}\right)$ (10) defined by the residue $N=\operatorname{Res}_{q_{2}=1}\left(m_{2 \star}\right)$ along $q_{2}=1$ matches with the filtration

$$
0 \subset \pi^{*}\left(\operatorname{Ker} r^{*}\right) \subset \operatorname{Im} \pi^{*} \subset H^{*}\left(X_{\text {res }}\right) .
$$

The quantum products of elements in $\operatorname{Im} \pi^{*}$ are regular at $q_{2}=1$ and the map

$$
r^{*} \circ\left(\pi^{*}\right)^{-1}: \operatorname{Im} \pi^{*} \rightarrow H^{*}(\operatorname{Gr}(2,4))
$$

intertwines the quantum product $\left.\star\right|_{q_{2}=1}$ on $\operatorname{Im} \pi^{*}=V$ with the quantum product on $H^{*}(\operatorname{Gr}(2,4))$ under the identification $q_{1}=q$ of the Novikov variables. This map also preserves the Poincaré pairing. 
Remark 5.3. Since $N$ is self-adjoing with respect to the Poincaré pairing, we have $(\operatorname{Ker} N)^{\perp}=\operatorname{Im} N$. Thus the Poincaré pairing induces a non-degenerate pairing on $V / W=\operatorname{Ker} N /\left(\operatorname{Ker} N \cap(\operatorname{Ker} N)^{\perp}\right)$.

In the above theorem, we identified the subquotient $\left(V / W,\left.\star\right|_{q_{2}=1}\right)$ of $H^{*}\left(X_{\text {res }}\right)$ with a subring of the quantum cohomology of $\operatorname{Gr}(2,4)$. We can extend this isomorphism to the whole of $H^{*}(\operatorname{Gr}(2,4))$ as follows. The weight filtration $W_{-2} \subset W_{-1} \subset W_{0} \subset W_{1} \subset$ $W_{2}=H^{\text {even }}\left(X_{\text {res }}\right)$ associated to the nilpotent endomorphism $N$ (see e.g. [3, A.2]) is given as follows:

$$
\begin{aligned}
& W_{-2}=W_{-1}=\operatorname{Span}\left\{m_{1}^{3}-2 m_{1}^{2} m_{2}\right\} \\
& W_{0}=W_{1}=\operatorname{Span}\left\{m_{1}^{3}-2 m_{1}^{2} m_{2}, m_{1}^{2}-2 m_{1} m_{2}, 1, m_{1}, m_{1}^{2}, m_{1}^{3}, m_{1}^{4}\right\} .
\end{aligned}
$$

This is illustrated by the following table:

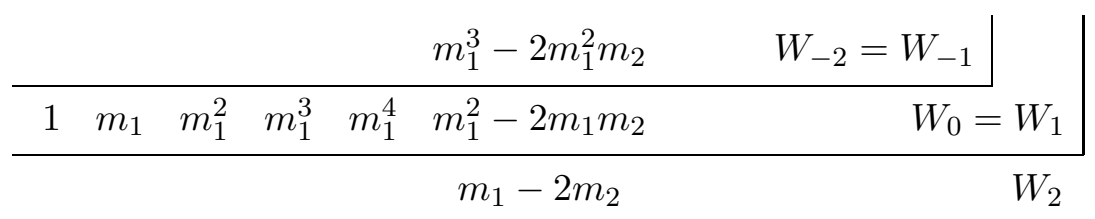

Therefore $V / W$ can be regarded as a subspace of $W_{0} / W_{-1}$. We define a linear isomorphism $\theta: W_{0} / W_{-1} \cong H^{*}(\operatorname{Gr}(2,4))$ by

$$
\begin{aligned}
\theta\left(m_{1}^{i}\right) & =d^{i} \quad \text { for } 0 \leq i \leq 4, \\
\theta\left(m_{1}^{2}-2 m_{1} m_{2}\right) & =\sqrt{-1}\left(d^{2}-2 \delta\right) .
\end{aligned}
$$

This gives an extension of the map $r^{*} \circ\left(\pi^{*}\right)^{-1}: V / W \rightarrow H^{*}(\operatorname{Gr}(2,4))$. We have the following:

Theorem 5.4. The quantum products of elements in $W_{0}$ are regular at $q_{2}=1$ and belong to $W_{0}$. The quantum product $\left.\star\right|_{q_{2}=1}$ on $W_{0}$ descends to $W_{0} / W_{-1}$ and $\theta$ induces an isomorphism of rings:

$$
\theta:\left(W_{0} / W_{-1},\left.\star\right|_{q_{2}=1}\right) \cong\left(H^{*}(\operatorname{Gr}(2,4)), \star\right)
$$

under the identification $q_{1}=q$. Moreover $\theta$ preserves the Poincaré pairing.

Remark 5.5. It is curious that we have imaginary numbers in the isomorphism $\theta$. The assignment $\theta: m_{1}^{2}-2 m_{1} m_{2} \mapsto \sqrt{-1}\left(d^{2}-2 \delta\right)$ is uniquely determined up to sign if we require that $\theta$ coincides with $r^{*} \circ\left(\pi^{*}\right)^{-1}$ on $V / W$ and intertwines the quantum products.

\section{EXAmple: $\operatorname{Gr}(2,5)$}

In this section we study an extremal transition of the 6-dimenional Fano variety $\operatorname{Gr}(2,5)$, the space of complex two planes in $\mathbb{C}^{5}$. Unlike the previous two examples in $\oint_{4}$ and $\mathrm{s}$, the image of the Plücker embedding of $\operatorname{Gr}(2,5)$ is not a hypersurface nor a complete intersection. We use the toric degeneration of $\operatorname{Gr}(2,5)$ and its crepant resolution studied by Gonciulea-Lakshmibai [9] and Batyrev-Ciocan-Fontanine-Kimvan-Straten [1]. 
According to [9, 1], the Grassmannian $\operatorname{Gr}(2,5)$ admits a flat degeneration to the Gorenstein toric variety $X_{\text {sing }}$ defined by the following 6-dimensional fan. The primitive generators of the 1-dimensional cones are:

$$
\begin{array}{ll}
r_{1}=(1,0,0,0,0,0), \quad r_{2}=(-1,1,0,0,0,0), & r_{3}=(-1,0,1,0,0,0), \\
r_{4}=(0,-1,0,1,0,0), & r_{5}=(0,0,-1,1,0,0), \quad r_{6}=(0,0,-1,0,1,0), \\
r_{7}=(0,0,0,-1,0,1), \quad r_{8}=(0,0,0,0,-1,1), & r_{9}=(0,0,0,0,0,-1) .
\end{array}
$$

The top dimensional cones are:

$$
\begin{aligned}
& \left\langle r_{2}, r_{3}, r_{4}, r_{5}, r_{6}, r_{7}, r_{8}, r_{9}\right\rangle, \quad\left\langle r_{1}, r_{2}, r_{3}, r_{4}, r_{5}, r_{6}, r_{7}, r_{8}\right\rangle, \\
& \left\langle r_{1}, r_{4}, r_{5}, r_{6}, r_{7}, r_{8}, r_{9}\right\rangle, \quad\left\langle r_{1}, r_{2}, r_{5}, r_{6}, r_{7}, r_{8}, r_{9}\right\rangle, \quad\left\langle r_{1}, r_{2}, r_{3}, r_{4}, r_{5}, r_{8}, r_{9}\right\rangle, \\
& \left\langle r_{1}, r_{2}, r_{3}, r_{4}, r_{5}, r_{6}, r_{9}\right\rangle, \quad\left\langle r_{1}, r_{3}, r_{4}, r_{7}, r_{8}, r_{9}\right\rangle, \quad\left\langle r_{1}, r_{3}, r_{4}, r_{6}, r_{7}, r_{9}\right\rangle, \\
& \left\langle r_{1}, r_{2}, r_{3}, r_{7}, r_{8}, r_{9}\right\rangle, \quad\left\langle r_{1}, r_{2}, r_{3}, r_{6}, r_{7}, r_{9}\right\rangle
\end{aligned}
$$

In order to obtain a crepant small resolution of $X_{\text {sing }}$, we divide non-simplicial cones as follows:

- divide $\left\langle r_{2}, r_{3}, r_{4}, r_{5}, r_{6}, r_{7}, r_{8}, r_{9}\right\rangle$ into $\left\langle r_{2}, r_{3}, r_{5}, r_{6}, r_{7}, r_{9}\right\rangle,\left\langle r_{2}, r_{3}, r_{5}, r_{7}, r_{8}, r_{9}\right\rangle$, $\left\langle r_{3}, r_{4}, r_{5}, r_{6}, r_{7}, r_{9}\right\rangle,\left\langle r_{3}, r_{4}, r_{5}, r_{7}, r_{8}, r_{9}\right\rangle ;$

- divide $\left\langle r_{1}, r_{2}, r_{3}, r_{4}, r_{5}, r_{6}, r_{7}, r_{8}\right\rangle$ into $\left\langle r_{1}, r_{2}, r_{3}, r_{5}, r_{6}, r_{7}\right\rangle,\left\langle r_{1}, r_{2}, r_{3}, r_{5}, r_{7}, r_{8}\right\rangle$, $\left\langle r_{1}, r_{3}, r_{4}, r_{5}, r_{6}, r_{7}\right\rangle,\left\langle r_{1}, r_{3}, r_{4}, r_{5}, r_{7}, r_{8}\right\rangle$

- divide $\left\langle r_{1}, r_{4}, r_{5}, r_{6}, r_{7}, r_{8}, r_{9}\right\rangle$ into $\left\langle r_{1}, r_{4}, r_{5}, r_{6}, r_{7}, r_{9}\right\rangle,\left\langle r_{1}, r_{4}, r_{5}, r_{7}, r_{8}, r_{9}\right\rangle$;

- divide $\left\langle r_{1}, r_{2}, r_{5}, r_{6}, r_{7}, r_{8}, r_{9}\right\rangle$ into $\left\langle r_{1}, r_{2}, r_{5}, r_{6}, r_{7}, r_{9}\right\rangle,\left\langle r_{1}, r_{2}, r_{5}, r_{7}, r_{8}, r_{9}\right\rangle$;

- divide $\left\langle r_{1}, r_{2}, r_{3}, r_{4}, r_{5}, r_{8}, r_{9}\right\rangle$ into $\left\langle r_{1}, r_{2}, r_{3}, r_{5}, r_{8}, r_{9}\right\rangle,\left\langle r_{1}, r_{3}, r_{4}, r_{5}, r_{8}, r_{9}\right\rangle$;

- divide $\left\langle r_{1}, r_{2}, r_{3}, r_{4}, r_{5}, r_{6}, r_{9}\right\rangle$ into $\left\langle r_{1}, r_{2}, r_{3}, r_{5}, r_{6}, r_{9}\right\rangle,\left\langle r_{1}, r_{3}, r_{4}, r_{5}, r_{6}, r_{9}\right\rangle$.

These subdivisions define a smooth toric variety $X_{\text {res }}$. In this section we study a relationship between the quantum cohomology of $\operatorname{Gr}(2,5)$ and $X_{\text {res }}$.

6.1. Quantum cohomology of $\operatorname{Gr}(2,5)$. We refer the reader to [2, 国 for the quatnum cohomology of $\operatorname{Gr}(2,5)$. It is well known that the Poincaré duals of the Schubert cycles form an additive basis of the cohomology ring of $\operatorname{Gr}(2,5)$. Fix a full flag $0 \subset F_{1} \subset F_{2} \subset$ $\cdots \subset F_{5}=\mathbb{C}^{5}$. The Schubert cycle $\Omega_{\left(a_{1}, a_{2}\right)} \subset \operatorname{Gr}(2,5)$, indexed by a pair $\left(a_{1}, a_{2}\right)$ of integers satisfying $3 \geq a_{1} \geq a_{2} \geq 0$, is given by:

$$
\Omega_{\left(a_{1}, a_{2}\right)}=\left\{V \subset \mathbb{C}^{5}: \operatorname{dim} V=2, \operatorname{dim}\left(V \cap F_{4-a_{1}}\right) \geq 1, V \subset F_{5-a_{2}}\right\} .
$$

We denote by $\omega_{\left(a_{1}, a_{2}\right)} \in H^{2\left(a_{1}+a_{2}\right)}(\operatorname{Gr}(2,5))$ the Poincaré dual of the Schubert cycle $\Omega_{\left(a_{1}, a_{2}\right)}$. The dual basis of $\left\{\omega_{\left(a_{1}, a_{2}\right)}\right\}$ is given by $\left\{\omega_{\left(3-a_{2}, 3-a_{1}\right)}\right\}$. We choose the following additive basis of $H^{*}(\operatorname{Gr}(2,5))$ :

$$
\left\{\omega_{(0,0)}, \omega_{(1,0)}, \omega_{(1,1)}, \omega_{(2,0)}, \omega_{(2,1)}, \omega_{(3,0)}, \omega_{(3,1)}, \omega_{(2,2)}, \omega_{(3,2)}, \omega_{(3,3)}\right\} .
$$

Let $q$ be the Novikov variable dual to the ample class $\omega_{(1,0)} \in H^{2}(\operatorname{Gr}(2,5))$. We have $\operatorname{deg} q=10$. The class $\omega_{(1,0)}$ generates the small quantum cohomology ring of $\operatorname{Gr}(2,5)$ 
and its quantum product is given by the following matrix:

$$
\omega_{(1,0) \star}=\left(\begin{array}{cccccccccc}
0 & 0 & 0 & 0 & 0 & 0 & q & 0 & 0 & 0 \\
1 & 0 & 0 & 0 & 0 & 0 & 0 & 0 & q & 0 \\
0 & 1 & 0 & 0 & 0 & 0 & 0 & 0 & 0 & 0 \\
0 & 1 & 0 & 0 & 0 & 0 & 0 & 0 & 0 & q \\
0 & 0 & 1 & 1 & 0 & 0 & 0 & 0 & 0 & 0 \\
0 & 0 & 0 & 1 & 0 & 0 & 0 & 0 & 0 & 0 \\
0 & 0 & 0 & 0 & 1 & 1 & 0 & 0 & 0 & 0 \\
0 & 0 & 0 & 0 & 1 & 0 & 0 & 0 & 0 & 0 \\
0 & 0 & 0 & 0 & 0 & 0 & 1 & 1 & 0 & 0 \\
0 & 0 & 0 & 0 & 0 & 0 & 0 & 0 & 1 & 0
\end{array}\right) .
$$

6.2. Quantum cohomology of $X_{\text {res }}$. Let $R_{i}$ denote the class of the toric divisor corresponding to the ray $\mathbb{R}_{\geq 0} r_{i}$. We choose a basis $\left\{m_{1}, m_{2}, m_{3}\right\}$ of $H^{2}\left(X_{\text {res }}\right)$ as $m_{1}=$ $R_{1}, m_{2}=R_{2}, m_{3}=R_{6}$. Then we have

$$
\begin{aligned}
& R_{1}=m_{1}, R_{2}=m_{2}, R_{3}=m_{1}-m_{2}, R_{4}=m_{2}, R_{5}=m_{1}-m_{2}-m_{3}, \\
& R_{6}=m_{3}, R_{7}=m_{1}-m_{3}, R_{8}=m_{3}, R_{9}=m_{1} .
\end{aligned}
$$

The cohomology ring of $X_{\text {res }}$ is given by:

$$
H^{\star}\left(X_{\mathrm{sm}}\right)=\mathbb{C}\left[m_{1}, m_{2}, m_{3}\right] /\left\langle m_{2}^{2}, m_{3}^{2}, m_{1}^{2}\left(m_{1}-m_{2}\right)\left(m_{1}-m_{2}-m_{3}\right)\left(m_{1}-m_{3}\right)\right\rangle .
$$

The classes $m_{1}, m_{2}, m_{3}$ span the nef cone of $X_{\text {res }}$. Let $\left\{\beta_{1}, \beta_{2}, \beta_{3}\right\} \subset H_{2}\left(X_{\text {res }}\right)$ be the dual basis of $\left\{m_{1}, m_{2}, m_{3}\right\}$; they span the Mori cone of $X_{\text {res }}$. For $d=n_{1} \beta_{1}+n_{2} \beta_{2}+$ $n_{3} \beta_{3} \in H_{2}\left(X_{\text {res }}\right)$, we write $q^{d}=q_{1}^{n_{1}} q_{2}^{n_{2}} q_{3}^{n_{3}}$, where $q_{i}=q^{\beta_{i}}$. We have $\operatorname{deg} q_{1}=10$, $\operatorname{deg} q_{2}=\operatorname{deg} q_{3}=0$. We choose the following basis for $H^{*}\left(X_{\text {res }}\right)$ :

$$
\left\{\begin{array}{l}
1, m_{1}, m_{2}, m_{3}, m_{1}^{2}, m_{1} m_{2}, m_{1} m_{3}, m_{2} m_{3}, m_{1}^{3}, m_{1}^{2} m_{2}, m_{1}^{2} m_{3}, \\
m_{1} m_{2} m_{3}, m_{1}^{4}, m_{1}^{3} m_{2}, m_{1}^{3} m_{3}, m_{1}^{2} m_{2} m_{3}, m_{1}^{5}, m_{1}^{4} m_{2}, m_{1}^{4} m_{3}, m_{1}^{6}
\end{array}\right\}
$$

We use Givental's mirror theorem [7] to calculate the quantum product; see Appendix A for the details.

The quantum products of $m_{1}$ with cohomology classes in the chosen basis (12) are as follows:

$$
\begin{aligned}
m_{1} \star m_{1}^{4}= & m_{1}^{5}+q_{1}\left(1+q_{2}+q_{3}\right), \\
m_{1} \star m_{1}^{3} m_{2}= & m_{1}^{4} m_{2}+q_{1} q_{2}, \\
m_{1} \star m_{1}^{3} m_{3}= & m_{1}^{4} m_{3}+q_{1} q_{3}, \\
m_{1} \star m_{1}^{5}= & m_{1}^{6}+\left(2 m_{2}+2 m_{3}\right) q_{1}+\left(m_{3}+2 m_{1}-2 m_{2}\right) q_{1} q_{2} \\
& +\left(m_{2}+2 m_{1}-2 m_{3}\right) q_{1} q_{3}+\left(m_{1}-m_{2}-m_{3}\right) q_{1} q_{2} q_{3}, \\
m_{1} \star m_{1}^{4} m_{2}= & m_{1}^{5} m_{2}+m_{2} q_{1}+\left(m_{1}-m_{2}+m_{3}\right) q_{1} q_{2}+m_{2} q_{1} q_{3}+\left(m_{1}-m_{2}-m_{3}\right) q_{1} q_{2} q_{3}, \\
m_{1} \star m_{1}^{4} m_{3}= & m_{1}^{5} m_{3}+m_{3} q_{1}+\left(m_{1}-m_{3}+m_{2}\right) q_{1} q_{3}+m_{3} q_{1} q_{2}+\left(m_{1}-m_{2}-m_{3}\right) q_{1} q_{2} q_{3}, \\
m_{1} \star m_{1}^{6}= & 5 m_{2} m_{3} q_{1}+\left(5 m_{1} m_{3}-5 m_{2} m_{3}\right) q_{1} q_{2}+\left(5 m_{1} m_{2}-5 m_{2} m_{3}\right) q_{1} q_{3}
\end{aligned}
$$

and all the other quantum products coincide with the cup products. 
The quantum products of $m_{2}$ with cohomology classes in the chosen basis (12) are as follows:

$$
\begin{aligned}
m_{2} \star m_{2}= & \left(m_{1}-m_{2}\right)\left(m_{1}-m_{2}-m_{3}\right) \frac{q_{2}}{1-q_{2}}, \\
m_{2} \star m_{1} m_{2}= & m_{1}\left(m_{1}-m_{2}\right)\left(m_{1}-m_{2}-m_{3}\right) \frac{q_{2}}{1-q_{2}}, \\
m_{2} \star m_{2} m_{3}= & m_{3}\left(m_{1}-m_{2}\right)\left(m_{1}-m_{2}-m_{3}\right) \frac{q_{2}}{1-q_{2}} \\
& -\left(m_{1}-m_{2}\right)\left(m_{1}-m_{3}\right)\left(m_{1}-m_{2}-m_{3}\right) \frac{q_{2} q_{3}}{\left(1-q_{2}\right)\left(1-q_{2}-q_{3}\right)}, \\
m_{2} \star m_{1}^{2} m_{2}= & m_{1}^{2}\left(m_{1}-m_{2}\right)\left(m_{1}-m_{2}-m_{3}\right) \frac{q_{2}}{1-q_{2}}, \\
m_{2} \star m_{1} m_{2} m_{3}= & m_{1} m_{3}\left(m_{1}-m_{2}\right)\left(m_{1}-m_{2}-m_{3}\right) \frac{q_{2}}{1-q_{2}} \\
& -m_{1}\left(m_{1}-m_{2}\right)\left(m_{1}-m_{3}\right)\left(m_{1}-m_{2}-m_{3}\right) \frac{q_{2} q_{3}}{\left(1-q_{2}\right)\left(1-q_{2}-q_{3}\right)} \\
m_{2} \star m_{1}^{4}= & m_{1}^{4} m_{2}+q_{1} q_{2}, \\
m_{2} \star m_{1}^{3} m_{2}= & m_{1}^{3}\left(m_{1}-m_{2}\right)\left(m_{1}-m_{2}-m_{3}\right) \frac{q_{2}}{1-q_{2}}+q_{1} q_{2}, \\
m_{2} \star m_{1}^{2} m_{2} m_{3}= & m_{1}^{2} m_{3}\left(m_{1}-m_{2}\right)\left(m_{1}-m_{2}-m_{3}\right) \frac{q_{2}}{1-q_{2}}, \\
m_{2} \star m_{1}^{5}= & m_{1}^{5} m_{2}+\left(m_{3}+2 m_{1}-2 m_{2}\right) q_{1} q_{2}+\left(m_{1}-m_{2}-m_{3}\right) q_{1} q_{2} q_{3}, \\
m_{2} \star m_{1}^{4} m_{2}= & \left(m_{1}-m_{2}+m_{3}\right) q_{1} q_{2}+\left(m_{1}-m_{2}-m_{3}\right) q_{1} q_{2} q_{3}, \\
m_{2} \star m_{1}^{4} m_{3}= & m_{1}^{4} m_{2} m_{3}+\left(m_{1}-m_{2}-m_{3}\right) q_{1} q_{2} q_{3}+m_{3} q_{1} q_{2}, \\
m_{2} \star m_{1}^{6}= & \left(5 m_{1} m_{3}-5 m_{2} m_{3}\right) q_{1} q_{2}
\end{aligned}
$$

All the other quantum products with $m_{2}$ are the same as the cup products.

The quantum products of $m_{3}$ with cohomology classes in the chosen basis (12) are as follows:

$$
\begin{aligned}
m_{3} \star m_{3}= & \left(m_{1}-m_{3}\right)\left(m_{1}-m_{2}-m_{3}\right) \frac{q_{3}}{1-q_{3}}, \\
m_{3} \star m_{1} m_{3}= & m_{1}\left(m_{1}-m_{3}\right)\left(m_{1}-m_{2}-m_{3}\right) \frac{q_{3}}{1-q_{3}}, \\
m_{3} \star m_{2} m_{3}= & m_{2}\left(m_{1}-m_{3}\right)\left(m_{1}-m_{2}-m_{3}\right) \frac{q_{3}}{1-q_{3}}, \\
& -\left(m_{1}-m_{2}\right)\left(m_{1}-m_{3}\right)\left(m_{1}-m_{2}-m_{3}\right) \frac{q_{2} q_{3}}{\left(1-q_{3}\right)\left(1-q_{2}-q_{3}\right)}, \\
m_{3} \star m_{1}^{2} m_{3}= & m_{1}^{2}\left(m_{1}-m_{3}\right)\left(m_{1}-m_{2}-m_{3}\right) \frac{q_{3}}{1-q_{3}}, \\
m_{3} \star m_{1} m_{2} m_{3}= & m_{1} m_{2}\left(m_{1}-m_{3}\right)\left(m_{1}-m_{2}-m_{3}\right) \frac{q_{3}}{1-q_{3}}, \\
& -m_{1}\left(m_{1}-m_{2}\right)\left(m_{1}-m_{3}\right)\left(m_{1}-m_{2}-m_{3}\right) \frac{q_{2} q_{3}}{\left(1-q_{3}\right)\left(1-q_{2}-q_{3}\right)}, \\
m_{3} \star m_{1}^{4}= & m_{1}^{4} m_{3}+q_{1} q_{3},
\end{aligned}
$$




$$
\begin{aligned}
m_{3} \star m_{1}^{3} m_{3} & =m_{1}^{3}\left(m_{1}-m_{3}\right)\left(m_{1}-m_{2}-m_{3}\right) \frac{q_{3}}{1-q_{3}}+q_{1} q_{3}, \\
m_{3} \star m_{1}^{2} m_{2} m_{3} & =m_{1}^{2} m_{2}\left(m_{1}-m_{3}\right)\left(m_{1}-m_{2}-m_{3}\right) \frac{q_{3}}{1-q_{3}}, \\
m_{3} \star m_{1}^{5} & =m_{1}^{5} m_{3}+\left(m_{2}+2 m_{1}-2 m_{3}\right) q_{1} q_{3}+\left(m_{1}-m_{2}-m_{3}\right) q_{1} q_{2} q_{3}, \\
m_{3} \star m_{1}^{4} m_{2} & =m_{1}^{4} m_{2} m_{3}+m_{2} q_{1} q_{3}+\left(m_{1}-m_{2}-m_{3}\right) q_{1} q_{2} q_{3}, \\
m_{3} \star m_{1}^{4} m_{3} & =\left(m_{1}-m_{3}+m_{2}\right) q_{1} q_{3}+\left(m_{1}-m_{2}-m_{3}\right) q_{1} q_{2} q_{3}, \\
m_{3} \star m_{1}^{6} & =\left(5 m_{1} m_{2}-5 m_{2} m_{3}\right) q_{1} q_{3}
\end{aligned}
$$

All the other quantum products with $m_{3}$ are the same as the cup products.

6.3. Comparison of quantum cohomology. The quantum product of $m_{2}$ has simple poles along $q_{2}=1$ and $q_{2}+q_{3}=1$; the quantum product of $m_{3}$ has simple poles along $q_{3}=1$ and $q_{2}+q_{3}=1$. We define

$$
\begin{aligned}
& N_{2}:=\left.\operatorname{Res}_{q_{2}=1}\left(m_{2} \star\right) \frac{d q_{2}}{q_{2}}\right|_{\left(q_{2}, q_{3}\right)=(1,1)} \\
& N_{3}:=\left.\operatorname{Res}_{q_{3}=1}\left(m_{3} \star\right) \frac{d q_{3}}{q_{3}}\right|_{\left(q_{2}, q_{3}\right)=(1,1)}
\end{aligned}
$$

These are nilpotent endomorphisms. Thus the monodromy of the quantum connection around the normal crossing divisors $\left(q_{2}=1\right),\left(q_{3}=1\right)$ is unipotent. As before, the endomorphisms $N_{2}, N_{3}$ define the filtration $0 \subset W \subset V \subset H^{*}\left(X_{\text {res }}\right)$ by:

$$
V:=\operatorname{Ker}\left(N_{2}\right) \cap \operatorname{Ker}\left(N_{3}\right), \quad W:=V \cap\left(\operatorname{Im}\left(N_{2}\right)+\operatorname{Im}\left(N_{3}\right)\right) .
$$

We have $\operatorname{dim} V=12$ and $\operatorname{dim} W=2$. The basis of $V$ is given by

$$
1, m_{1}, m_{1}^{2}, m_{1}^{3}, m_{1}^{4}, m_{1}^{5}, m_{1}^{6}, \alpha, m_{1} \alpha, m_{1}^{2} \alpha, m_{1}^{4} m_{2}, m_{1}^{4} m_{3},
$$

where $\alpha:=m_{1} m_{2}+m_{1} m_{3}-m_{2} m_{3}$, and the basis of $W$ is given by

$$
m_{1}^{4} m_{2}-m_{1}^{4} m_{3}, \quad 2 m_{1}^{5}-5 m_{1}^{4} m_{2} .
$$

Define a linear map $\theta: V \rightarrow H^{*}\left(X_{\mathrm{sm}}\right)$ as follows:

$$
\begin{aligned}
\theta\left(m_{1}^{i}\right) & =\left(\omega_{(1,0)}\right)^{i} & & 0 \leq i \leq 6, \\
\theta\left(m_{1}^{i} \alpha\right) & =\left(\omega_{(1,0)}\right)^{i} \omega_{(2,0)}, & & 0 \leq i \leq 2 \\
\theta\left(m_{1}^{4} m_{2}\right) & =2 \omega_{(3,2)} & & \\
\theta\left(m_{1}^{4} m_{3}\right) & =2 \omega_{(3,2)} . & &
\end{aligned}
$$

We have $\operatorname{Ker} \theta=W$ and the map $\theta$ induces an isomorphism:

$$
\theta: V / W \cong H^{*}(\operatorname{Gr}(2,5))
$$

Note that the quantum product of $m_{1}$ is regular along $q_{2}=q_{3}=1$. Since $\left(m_{1} \star\right)$ commutes with $\left(m_{2} \star\right)$ and $\left(m_{3} \star\right)$, it follows that $\left.\left(m_{1} \star\right)\right|_{q_{2}=q_{3}=1}$ commutes with $N_{2}$ and $N_{3}$; thus $\left.\left(m_{1} \star\right)\right|_{q_{2}=q_{3}=1}$ descends to the quotient space $V / W$ and defines a ring structure on $V / W$. The following result follows by a direct computation: 
Theorem 6.1. The quantum product on $H^{*}\left(X_{\mathrm{res}}\right)$ at $q_{2}=q_{3}=1$ descends to a welldefined product structure on $V / W$. The linear isomorphism $\theta: V / W \cong H^{*}(\operatorname{Gr}(2,5))$ intertwines the quantum product $\left.\star\right|_{q_{2}=q_{3}=1}$ on $V / W$ with the quantum product on $H^{*}(\mathrm{Gr}(2,5))$. Moreover $\theta$ preserves the Poincaré pairing.

Remark 6.2. When $a \neq 0$ and $b \neq 0$, the nilpotent operator $N=a N_{2}+b N_{3}$ defines a weight filtration $\left\{W_{\bullet}\right\}$ independent of $(a, b)$. The Jordan normal form of $N$ consists of 10 Jordan blocks of size 1 (one-by-one zero matrices) and 2 Jordan blocks of size 5 . Therefore $W_{0} / W_{-1}$ gives a 12 -dimensional space which is bigger than $H^{*}\left(X_{\mathrm{sm}}\right)$. The above quotient $V / W$ corresponds to Jordan blocks of size 1.

6.4. Topology of the extremal transition of $\operatorname{Gr}(2,5)$. We study a relationship between the map $\theta$ in Theorem 6.1 and the maps on cohomology induced by the natural maps $X_{\text {res }} \rightarrow X_{\text {sing }} \leftarrow X_{\mathrm{sm}}=\operatorname{Gr}(2,5)$. In this section, we prove the following.

Theorem 6.3. Let $\pi: X_{\text {res }} \rightarrow X_{\text {sing }}$ and $r: X_{\mathrm{sm}}=\operatorname{Gr}(2,5) \rightarrow X_{\text {sing }}$ denote the natural maps associated to the extremal transition of $\operatorname{Gr}(2,5)$. Let $V, W$ be as given in (13). We have the following commutative diagram

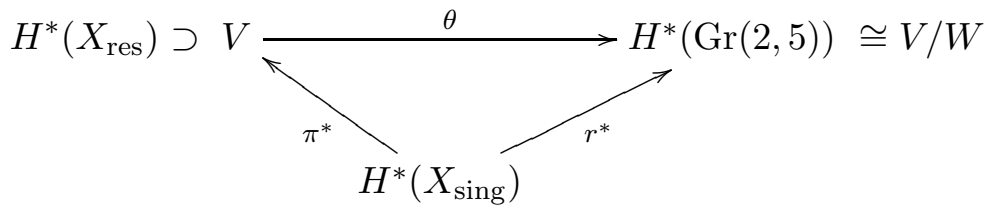

so that $\theta \circ \pi^{*}=r^{*}$, where $\theta$ is given in (14) and

(1) $\pi^{*}: H^{*}\left(X_{\text {sing }}\right) \rightarrow H^{*}\left(X_{\text {res }}\right)$ is injective and the image is contained in $V$;

(2) $r^{*}: H^{*}\left(X_{\text {sing }}\right) \rightarrow H^{*}\left(X_{\mathrm{sm}}\right)=H^{*}(\mathrm{Gr}(2,5))$ is neither injective nor surjective;

(3) $W \subset \operatorname{Im} \pi^{*} \varsubsetneqq V$ and $\pi^{*}\left(\operatorname{Ker} r^{*}\right)=W$.

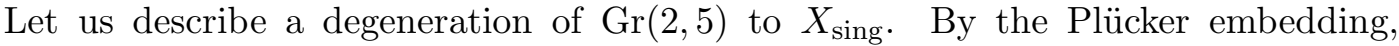
$\operatorname{Gr}(2,5)$ can be realized as the codimension 3 subvariety $X_{t} \subset \mathbb{P}^{9}$ (with $t \neq 0$ ) cut out by the following five equations:

$$
\begin{aligned}
& t Z_{12} Z_{34}-Z_{13} Z_{24}+Z_{14} Z_{23}=0 \\
& t Z_{12} Z_{35}-Z_{13} Z_{25}+Z_{15} Z_{23}=0 \\
& t Z_{12} Z_{45}-Z_{14} Z_{25}+Z_{15} Z_{24}=0 \\
& t Z_{13} Z_{45}-Z_{14} Z_{35}+Z_{15} Z_{34}=0 \\
& t Z_{23} Z_{45}-Z_{24} Z_{35}+Z_{25} Z_{34}=0
\end{aligned}
$$

where $\left(Z_{12}, Z_{13}, Z_{14}, Z_{15}, Z_{23}, Z_{24}, Z_{25}, Z_{34}, Z_{35}, Z_{45}\right)$ are homogeneous co-ordinates of $\mathbb{P}^{9}$. The central fiber $X_{0}$ gives the singular toric variety $X_{\text {sing. Let }} z_{1}, z_{2}, \ldots, z_{9}$ denote the homogeneous co-ordinates of the toric variety $X_{\text {sing }}$ corresponding to the toric divisors $R_{1}, \ldots, R_{9}$. Let $L=\mathcal{O}\left(R_{1}\right)$ be the line bundle on $X_{\text {sing }}$ corresponding to the Cartier toric divisor $R_{1}$. This line bundle $L$ defines an embedding of $X_{\text {sing }}$ into $\mathbb{P}^{9}$ via the following basis of $H^{0}\left(X_{\text {sing }}, L\right)$ :

$$
\begin{array}{llll}
Z_{12}=z_{1} & Z_{13}=z_{6} z_{7} & Z_{14}=z_{4} z_{5} z_{6} & Z_{15}=z_{2} z_{5} z_{6} \\
Z_{23}=z_{7} z_{8} & Z_{24}=z_{4} z_{5} z_{8} & Z_{25}=z_{2} z_{5} z_{8} & Z_{34}=z_{3} z_{4} \\
Z_{35}=z_{2} z_{3} & Z_{45}=z_{9} . & &
\end{array}
$$


The image of this embedding coincides with $X_{0}$.

We start with the computation of $H^{*}\left(X_{\text {sing }}\right)$. For a subset $\left\{i_{1}, \ldots, i_{k}\right\} \subset\{1,2, \ldots, 9\}$, we write

$$
V\left(i_{1}, \ldots, i_{k}\right) \subset X_{\text {sing }} \quad \text { or } \quad \tilde{V}\left(i_{1}, \ldots, i_{k}\right) \subset X_{\text {res }}
$$

for the closed toric subvarieties associated with the cone $\left\langle r_{i_{1}}, r_{i_{2}}, \ldots, r_{i_{k}}\right\rangle$. Let $E \subset X_{\text {res }}$ denote the exceptional set of the resolution $\pi: X_{\text {res }} \rightarrow X_{\text {sing }}$ and let $S \subset X_{\text {sing }}$ denote the singular locus. We have

$$
S=S_{1} \cup S_{2}, \quad E=E_{1} \cup E_{2}
$$

with $S_{1}=V(2,3,4,5), S_{2}=V(5,6,7,8), E_{1}=\widetilde{V}(3,5), E_{2}=\widetilde{V}(5,7)$ and

$$
\begin{array}{ll}
S_{1} \cong S_{2} \cong \mathbb{P}^{3}, & S_{1} \cap S_{2} \cong \mathbb{P}^{1}, \\
E_{1} \cong E_{2} \cong \mathbb{P}^{1} \times \mathrm{Bl}_{\mathbb{P}^{1}}\left(\mathbb{P}^{3}\right), & E_{1} \cap E_{2} \cong \mathbb{P}^{1} \times \mathbb{P}^{1} \times \mathbb{P}^{1} .
\end{array}
$$

Here $\mathrm{Bl}_{\mathbb{P}^{1}}\left(\mathbb{P}^{3}\right)$ denotes the blowup of $\mathbb{P}^{3}$ along a line $\mathbb{P}^{1}$. The toric variety $X_{\text {sing }}$ has transversely conifold $\{x y=z w\}$ singularities along the smooth locus of $S$. Since odd cohomology groups of $E_{i}, S_{i}, E_{1} \cap E_{2}, S_{1} \cap S_{2}$ vanish, the Mayler-Vietoris exact sequences give

$$
\begin{aligned}
& 0 \longrightarrow H^{*}(S) \longrightarrow H^{*}\left(S_{1}\right) \oplus H^{*}\left(S_{2}\right) \longrightarrow H^{*}\left(S_{1} \cap S_{2}\right) \longrightarrow 0 \\
& 0 \longrightarrow H^{*}(E) \longrightarrow H^{*}\left(E_{1}\right) \oplus H^{*}\left(E_{2}\right) \longrightarrow H^{*}\left(E_{1} \cap E_{2}\right) \longrightarrow 0
\end{aligned}
$$

and thus

$$
\begin{array}{ll}
H^{*}(S)=\mathbb{C}, 0, \mathbb{C}, 0, \mathbb{C}^{2}, 0, \mathbb{C}^{2} & \text { for } *=0,1,2,3,4,5,6 \\
H^{*}(E)=\mathbb{C}, 0, \mathbb{C}^{3}, 0, \mathbb{C}^{5}, 0, \mathbb{C}^{5}, 0, \mathbb{C}^{2} & \text { for } *=0,1,2,3,4,5,6,7,8 .
\end{array}
$$

Lemma 6.4. The relative cohomology group of the pair $\left(X_{\mathrm{res}}, E\right)$ is given by the following table.

\begin{tabular}{c|ccccccccccccc} 
degree $p$ & 0 & 1 & 2 & 3 & 4 & 5 & 6 & 7 & 8 & 9 & 10 & 11 & 12 \\
\hline$H^{p}\left(X_{\text {res }}, E\right)$ & 0 & 0 & 0 & 0 & 0 & $\mathbb{C}$ & 0 & $\mathbb{C}$ & $\mathbb{C}^{2}$ & 0 & $\mathbb{C}^{3}$ & 0 & $\mathbb{C}$
\end{tabular}

The relative cohomology $H^{*}\left(X_{\mathrm{sing}}, S\right) \cong H^{*}\left(X_{\mathrm{res}}, E\right)$ is given by the same table.

Proof. This follows from the relative cohomology exact sequence associated with the pair $\left(X_{\text {res }}, E\right)$. Since the odd cohomology groups of $X_{\text {res }}$ and $E$ vanish, we have the exact sequence

$$
0 \longrightarrow H^{2 k}\left(X_{\mathrm{res}}, E\right) \longrightarrow H^{2 k}\left(X_{\mathrm{res}}\right) \longrightarrow H^{2 k}(E) \longrightarrow H^{2 k+1}\left(X_{\mathrm{res}}, E\right) \longrightarrow 0
$$

for each integer $k$. It suffices to study the restriction map $H^{2 k}\left(X_{\text {res }}\right) \rightarrow H^{2 k}(E)$. Since the spaces $X_{\text {res }}, E$ are toric, this can be done by standard methods: we find that

- $H^{0}\left(X_{\mathrm{res}}\right) \rightarrow H^{0}(E), H^{2}\left(X_{\mathrm{res}}\right) \rightarrow H^{2}(E)$ are isomorphisms;

- $H^{4}\left(X_{\text {res }}\right) \rightarrow H^{4}(E), H^{6}\left(X_{\text {res }}\right) \rightarrow H^{6}(E)$ are injective;

- $H^{8}\left(X_{\text {res }}\right) \rightarrow H^{8}(E)$ is surjective.

The conclusion follows.

Lemma 6.5. The cohomology group of $X_{\text {sing }}$ is given by the following table.

\begin{tabular}{l|ccccccccccccc} 
degree $p$ & 0 & 1 & 2 & 3 & 4 & 5 & 6 & 7 & 8 & 9 & 10 & 11 & 12 \\
\hline$H^{p}\left(X_{\text {sing }}\right)$ & $\mathbb{C}$ & 0 & $\mathbb{C}$ & 0 & $\mathbb{C}$ & 0 & $\mathbb{C}$ & 0 & $\mathbb{C}^{2}$ & 0 & $\mathbb{C}^{3}$ & 0 & $\mathbb{C}$
\end{tabular}


Moreover the map $\pi^{*}: H^{*}\left(X_{\text {sing }}\right) \rightarrow H^{*}\left(X_{\text {res }}\right)$ is injective and $\operatorname{Im} \pi^{*}$ has the following basis:

$$
1, m_{1}, m_{1}^{2}, m_{1}^{3}, m_{1}^{4}, m_{1}^{2} \alpha, m_{1}^{5}, m_{1}^{4} m_{2}, m_{1}^{4} m_{3}, m_{1}^{6}
$$

with $\alpha=m_{1} m_{2}+m_{1} m_{3}-m_{2} m_{3}$. In particular, we have $\operatorname{Im} \pi^{*} \varsubsetneqq V$.

Proof. The relative cohomology exact sequence for the pair $\left(X_{\text {sing }}, S\right)$ and the previous Lemma 6.4 give $H^{i}\left(X_{\text {sing }}\right) \cong H^{i}(S)$ for $i=0,1,2,3$, the exact sequences

$$
0 \longrightarrow H^{p}\left(X_{\text {sing }}\right) \longrightarrow H^{p}(S) \longrightarrow H^{p+1}\left(X_{\text {sing }}, S\right) \longrightarrow H^{p+1}\left(X_{\text {sing }}\right) \longrightarrow 0
$$

for $p=4,6$, and $H^{k}\left(X_{\text {sing }}, S\right) \cong H^{k}\left(X_{\text {sing }}\right)$ for $k=8,9,10,11,12$. To determine $H^{p}\left(X_{\text {sing }}\right)$ for $p=4,5,6,7$, we use naturality of the long exact sequence. We have the commutative diagram:

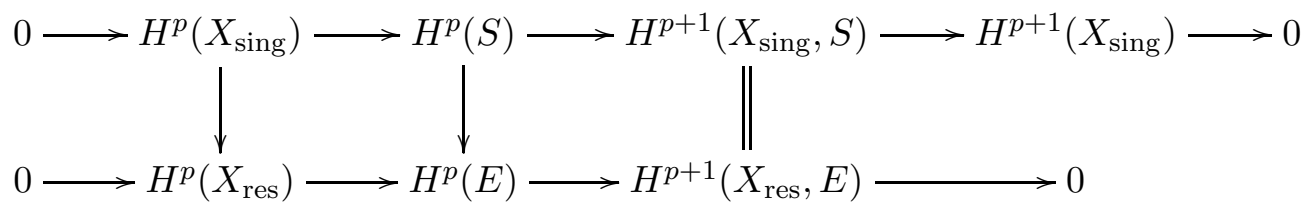

for $p=4,6$, where the rows are exact and the columns are induced by $\pi: X_{\text {res }} \rightarrow X_{\text {sing. }}$. For both $p=4$ and $p=6$, we can show that the images of the maps $H^{p}\left(X_{\text {res }}\right) \rightarrow$ $H^{p}(E)$ and $H^{p}(S) \rightarrow H^{p}(E)$ together span $H^{p}(E)$, and thus $H^{p}(S) \rightarrow H^{p+1}\left(X_{\text {sing }}, S\right)$ is surjective. The first statement follows.

To show the second statement, we note that the toric divisor $R_{1}$ is Cartier and ample on $X_{\text {sing. }}$. Therefore the class $m_{1}=R_{1}$ on $X_{\text {res }}$ lies in the image of $\pi^{*}: H^{2}\left(X_{\text {sing }}\right) \rightarrow$ $H^{2}\left(X_{\text {res }}\right)$. It follows that $m_{1}^{i}$ is a generator of $\pi^{*}\left(H^{2 i}\left(X_{\text {sing }}\right)\right) \cong \mathbb{C}$ for $i=1,2,3,6$. The image of the map $\pi^{*}: H^{8}\left(X_{\text {sing }}\right) \rightarrow H^{8}\left(X_{\text {res }}\right)$ can be computed via the commutative diagram:

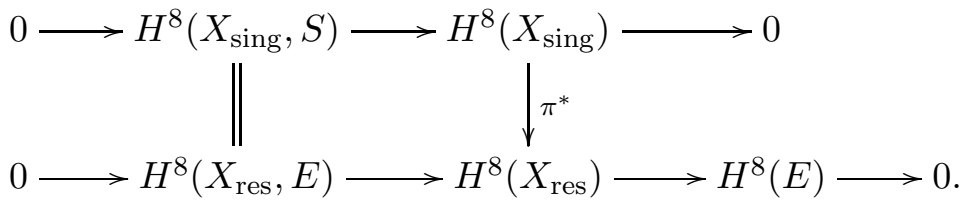

Therefore $\pi^{*}\left(H^{8}\left(X_{\text {sing }}\right)\right)$ equals the kernel of the restriction $H^{8}\left(X_{\text {res }}\right) \rightarrow H^{8}(E)$, and we can show that it is spanned by $m_{1}^{4}$ and $m_{1}^{2} \alpha$. By a similar argument, we find that $\pi^{*}: H^{10}\left(X_{\text {sing }}\right) \cong H^{10}\left(X_{\text {res }}\right)$. The conclusion follows.

Finally we compute the map $r^{*}: H^{*}\left(X_{\mathrm{sing}}\right) \rightarrow H^{*}\left(X_{\mathrm{sm}}\right)$.

Lemma 6.6. The map $r^{*} \circ\left(\pi^{*}\right)^{-1}: \operatorname{Im} \pi^{*} \rightarrow H^{*}\left(X_{\mathrm{sm}}\right)$ sends the basis of $\operatorname{Im} \pi^{*}$ given in Lemma 6.5 as follows:

$$
\begin{aligned}
m_{1}^{i} & \longmapsto \omega_{(1,0)}^{i} \quad 0 \leq i \leq 6, \\
m_{1}^{2} \alpha & \longmapsto \omega_{(1,0)}^{2} \omega_{(2,0)}, \\
m_{1}^{4} m_{2} & \longmapsto 2 \omega_{(3,2)}, \\
m_{1}^{4} m_{3} & \longmapsto 2 \omega_{(3,2)} .
\end{aligned}
$$


Proof. Abusing notation we write $m_{1}$ for the class of the Cartier toric divisor $R_{1}$ on $X_{\text {sing }}$, so that $\pi^{*}\left(m_{1}\right)=m_{1}$. Note that $m_{1} \in H^{2}\left(X_{\text {sing }}\right)$ or $\omega_{(1,0)} \in H^{2}\left(X_{\text {sm }}\right)$ is the restriction of the ample class $\mathcal{O}(1)$ on $\mathbb{P}^{9}$ to $X_{0}$ or to $X_{t}$ (with $t \neq 0$ ) respectively. Therefore $r^{*}$ sends $m_{1}$ to $\omega_{(1,0)}$. The images of $m_{1}^{4} m_{2}, m_{1}^{4} m_{3} \in \pi^{*}\left(H^{10}\left(X_{\text {sing }}\right)\right)$ under $r^{*} \circ\left(\pi^{*}\right)^{-1}$ can be easily computed from the commutative diagram:

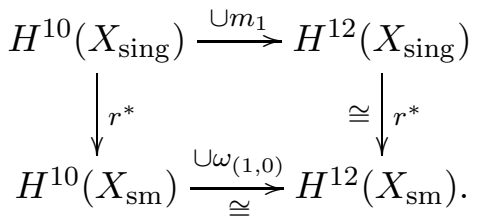

It remains to compute the image of $m_{1}^{2} \alpha \in \pi^{*}\left(H^{8}\left(X_{\text {sing }}\right)\right)$. By the commutative diagram

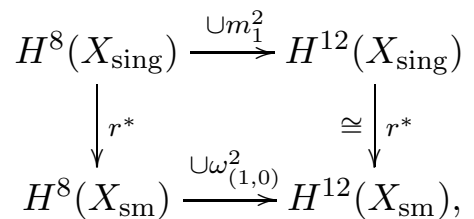

it follows that the kernel of $m_{1}^{2}: H^{8}\left(X_{\text {sing }}\right) \rightarrow H^{12}\left(X_{\text {sing }}\right)$ should be sent to the kernel of $\omega_{(1,0)}^{2}: H^{8}\left(X_{\mathrm{sm}}\right) \rightarrow H^{12}\left(X_{\mathrm{sm}}\right)$ under $r^{*}$. Therefore we have

$$
r^{*} \circ\left(\pi^{*}\right)^{-1}\left(3 m_{1}^{2}-5 m_{1}^{2} \alpha\right)=a\left(\omega_{(3,1)}-\omega_{(2,2)}\right)
$$

for some $a \in \mathbb{C}$. This implies $r^{*} \circ\left(\pi^{*}\right)^{-1}\left(m_{1}^{2} \alpha\right)=\frac{9-a}{5} \omega_{(3,1)}+\frac{6+a}{5} \omega_{(2,2)}$. To determine $a$, we use the fact $r_{*}\left[\Omega_{(1,1)}\right]=[V(2,9)]$ proved in Lemma 6.7 below. Since the map $\pi: \widetilde{V}(2,9) \rightarrow V(2,9)$ is birational, we have $\pi_{*}[\tilde{V}(2,9)]=[V(2,9)]$. Thus

$$
\begin{aligned}
m_{1}^{2} \alpha \cdot[\tilde{V}(2,9)] & =\left(\pi^{*}\right)^{-1}\left(m_{1}^{2} \alpha\right) \cdot[V(2,9)] \\
& =\left(r^{*} \circ\left(\pi^{*}\right)^{-1}\left(m_{1}^{2} \alpha\right)\right) \cdot\left[\Omega_{(1,1)}\right]=\frac{6+a}{5} .
\end{aligned}
$$

On the other hand, $m_{1}^{2} \alpha \cdot[\widetilde{V}(2,9)]=m_{1}^{2} \alpha R_{2} R_{9} \cdot\left[X_{\text {res }}\right]=1$. Therefore $a=-1$ and the conclusion follows.

Lemma 6.7. Consider the map $r_{*}: H_{8}\left(X_{\mathrm{sm}}\right)=H_{8}(\operatorname{Gr}(2,5)) \rightarrow H_{8}\left(X_{\text {sing }}\right)$ between homology groups. We have $r_{*}[\operatorname{Gr}(2,4)]=[V(2,9)]$, where $\operatorname{Gr}(2,4)$ is identified with the Schubert cycle $\Omega_{(1,1)}$ in $\operatorname{Gr}(2,5)$ (see (11)).

Proof. We consider the linear subspace

$$
\mathbb{P}^{5}=\left\{Z_{15}=Z_{25}=Z_{35}=Z_{45}=0\right\} \subset \mathbb{P}^{9}
$$

and restrict the family $X_{t}$ to $\mathbb{P}^{5}$. Note that $X_{t} \cap \mathbb{P}^{5}$ is defined by the equation $t Z_{12} Z_{34}-$ $Z_{13} Z_{24}+Z_{14} Z_{23}=0$ in $\mathbb{P}^{5}$. For $t \neq 0, X_{t} \cap \mathbb{P}^{5}$ is identified with the image of $\Omega_{(1,1)} \cong$ $\operatorname{Gr}(2,4)$ under the Plücker embedding. On the other hand, $X_{0} \cap \mathbb{P}^{5}$ is identified with

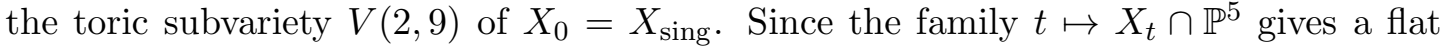
degeneration of $\operatorname{Gr}(2,4)$ to $V(2,9)$, the conclusion follows.

Theorem 6.3 follows easily from the computations in Lemmas 6.4, 6.5, 6.6. 


\section{Conjecture For partial flag varieties}

In this section we formulate a conjecture which describes the change of quantum cohomology under the extremal transition [1] of partial flag varieties. For a sequence of integers $0<n_{1}<n_{2}<\cdots<n_{l}<n$, we consider the partial flag variety:

$$
\operatorname{Fl}\left(n_{1}, n_{2}, \ldots, n_{l}, n\right)=\left\{V_{1} \subset V_{2} \subset \cdots \subset V_{l} \subset \mathbb{C}^{n}: \operatorname{dim} V_{i}=n_{i}\right\} .
$$

This space admits a flat degeneration to a Gorenstein Fano toric variety $X_{\text {sing }}$ and $X_{\text {sing }}$ has a small crepant resolution $X_{\text {res }}$.

We recall the toric varieties $X_{\text {sing }}, X_{\text {res }}$ from [1]. Let $D, S$ be the following subsets of $\mathbb{Z}^{2}$ :

$$
\begin{aligned}
D & =\bigcup_{p=1}^{l}\left\{(i, j) \in \mathbb{Z}^{2}: 0 \leq i \leq n-n_{p}-1,0 \leq j \leq n_{p}-1\right\}, \\
S & =\left\{\left(n-n_{1}, 0\right),\left(n-n_{2}, n_{1}\right), \ldots,\left(n-n_{l}, n_{l-1}\right),\left(0, n_{l}\right)\right\} .
\end{aligned}
$$

Elements of $D$ are called dots and elements of $S$ are called stars. Elements of $D \cup S$ form vertices of the ladder diagram [1], §2] which is an oriented graph. The set $E$ of oriented edges of the ladder diagram consists of pairs $e=(t(e), h(e))$ with $t(e), h(e) \in D \cup S$ such that $h(e)-t(e)=(1,0)$ or $h(e)-t(e)=(0,-1)$, where $t(e)$ is the tail and $h(e)$ is the head. Consider the vector space $\mathbb{R}^{D}$ with the standard basis $\left\{\mathbf{e}_{v}: v \in D\right\}$. We set $\mathbf{e}_{s}=0$ for $s \in S$. The fan $\Sigma_{\text {sing }}$ of the toric variety $X_{\text {sing }}$ is defined on $\mathbb{R}^{D}$; onedimensional cones of the fan are parametrized by $E$ and their primitive generators are given by

$$
r_{e}:=\mathbf{e}_{h(e)}-\mathbf{e}_{t(e)}
$$

for $e \in E$. The convex hull $\Delta \subset \mathbb{R}^{D}$ of the vectors $r_{e}, e \in E$ is a reflexive polytope [1], and the fan $\Sigma_{\text {sing }}$ is defined to be the set of cones over faces of $\Delta$. The fan $\Sigma_{\text {res }}$ of $X_{\text {res }}$ is given by a simplicial subdivision of $\Sigma_{\text {sing. For }} 1 \leq i \leq l$, a roof $\mathcal{R}_{i}$ is a collection of edges connecting the $(i+1)$ th $\operatorname{star}\left(n-n_{i+1}, n_{i}\right) \in S$ and the $i$ th $\operatorname{star}\left(n-n_{i}, n_{i-1}\right) \in S$ along the "boundary" of the ladder diagram (where we set $n_{0}=0, n_{l+1}=n$ ). More precisely,

$$
\begin{aligned}
\mathcal{R}_{i}= & \left\{\left(\left(n-n_{i+1}, n_{i}\right),\left(n-n_{i+1}, n_{i}-1\right)\right)\right\} \\
& \cup\left\{\left(\left(p, n_{i}-1\right),\left(p+1, n_{i}-1\right)\right): n-n_{i+1} \leq p \leq n-n_{i}-2\right\} \\
& \cup\left\{\left(\left(n-n_{i}-1, q\right),\left(n-n_{i}-1, q-1\right)\right): n_{i-1}+1 \leq q \leq n_{i}-1\right\} \\
& \cup\left\{\left(\left(n-n_{i}-1, n_{i-1}\right),\left(n-n_{i}, n_{i-1}\right)\right)\right\} .
\end{aligned}
$$

A box of the ladder diagram is a subset of 4 vertices of the form

$$
b=\{(i, j),(i+1, j),(i, j+1),(i+1, j+1)\} \subset D \cup S .
$$

The corner $\mathcal{C}_{b}$ of $b$ is the subset $\{((i, j+1),(i, j)),((i, j),(i+1, j))\}$ of edges adjacent to the lower left vertex $(i, j)$ of the box $b$. We write $\mathcal{C}_{b}^{-}$for the upper right corner $\{((i, j+1),(i+1, j+1)),((i+1, j+1),(i+1, j))\}$. Let Box denote the set of boxes of the ladder diagram. The fan $\Sigma_{\text {res }}$ of $X_{\text {res }}$ is a simplicial subdivision of $\Sigma_{\text {sing }}$ such that $\mathcal{R}_{1}, \ldots, \mathcal{R}_{l}$ and $\mathcal{C}_{b}$ with $b \in$ Box are primitive collections. Here we mean by a primitive collection a minimal subset $P$ of $E$ such that the cone spanned by $\left\{r_{e}: e \in P\right\}$ does 
not belong to the fan $\Sigma_{\text {res }}$. The corresponding toric variety $X_{\text {res }}$ gives a small crepant resolution of $X_{\text {sing }}$ [1, §3]. We write $\pi: X_{\text {res }} \rightarrow X_{\text {sing }}$ for the natural map.

The Mori cone of $X_{\mathrm{sm}}=\mathrm{Fl}\left(n_{1}, \ldots, n_{l}, n\right)$ is a simplicial cone generated by $\Delta_{i}$, where $\Delta_{i}$ is the class of a curve in the fiber of the natural map $\operatorname{Fl}\left(n_{1}, \ldots, n_{l}, n\right) \rightarrow$ $\mathrm{Fl}\left(n_{1}, \ldots, \widehat{n_{i}}, \ldots, n_{l}, n\right)$. We write $\bar{q}_{i}$ for the Novikov variable of $X_{\mathrm{sm}}$ corresponding to $\Delta_{i}$ for $1 \leq i \leq l$. The Mori cone of $X_{\text {res }}$ is also a simplicial cone generated by the curve classes $C_{i}$ with $1 \leq i \leq l$ and $C_{b}$ with $b \in$ Box [11, §3], where $C_{i}$ is defined by the "roof relation" $\sum_{e \in \mathcal{R}_{i}} r_{e}=0$ and $C_{b}$ is defined by the "box relation" $\sum_{e \in \mathcal{C}_{b}} r_{e}-\sum_{e \in \mathcal{C}_{b}^{-}} r_{e}=0$. We write $q_{i}, q_{b}$ for the Novikov variables corresponding to $C_{i}, C_{b}$. The morphism $\pi: X_{\text {res }} \rightarrow X_{\text {sing }}$ contracts the extremal rays $\mathbb{R}_{\geq 0} C_{b}$ with $b \in$ Box. We write $\phi_{i}$ with $1 \leq i \leq l$ and $\phi_{b}$ with $b \in \operatorname{Box}$ for the basis of $H^{2}\left(X_{\text {res }}\right)$ dual to $C_{i}, C_{b}$. We also write $\bar{\phi}_{i}$ with $1 \leq i \leq l$ for the basis of $H^{2}\left(X_{\mathrm{sm}}\right)$ dual to $\Delta_{i}$.

Conjecture 7.1. Let $X_{\mathrm{sm}}=\mathrm{Fl}\left(n_{1}, \ldots, n_{l}, n\right), X_{\mathrm{sing}}, X_{\mathrm{res}}$ be as above.

(1) The structure constants of the small quantum product of $X_{\text {res }}$ are polynomials in $q_{1}, \ldots, q_{l}$ with coefficients in rational functions of $q_{b}, b \in$ Box.

(2) The small quantum connection of $X_{\mathrm{res}}$ has logarithmic singularities along the normal crossing divisor $\prod_{b \in \mathrm{Box}}\left(q_{b}-1\right)=0$, and the residue endomorphisms along $q_{b}=1$ (with $b \in \mathrm{Box}$ ) are nilpotent. More precisely, $\left(\phi_{i} \star\right)$ with $1 \leq i \leq l$ is regular along $\Delta_{\mathrm{exc}}:=\left\{q_{b}=1(\forall b \in \mathrm{Box})\right\},\left(\phi_{b} \star\right)$ with $b \in \operatorname{Box}$ has simple poles along $\left\{q_{b}=1\right\}$ but no poles along $\left\{q_{b^{\prime}}=1\right\}$ for $b^{\prime} \neq b$, and

$$
N_{b}:=\left.\operatorname{Res}_{q_{b}=1}\left(\phi_{b} \star\right) \frac{d q_{b}}{q_{b}}\right|_{\Delta_{\text {exc }}}
$$

is a nilpotent endomorphism which does not depend on $q_{1}, \ldots, q_{l}$.

(3) Define a filtration $0 \subset W \subset V \subset H^{*}\left(X_{\text {res }}\right)$ by $V=\bigcap_{b \in \operatorname{Box}} \operatorname{Ker} N_{b}$ and $W=$ $V \cap \sum_{b \in \text { Box }} \operatorname{Im} N_{b}$. Along the locus $\Delta_{\mathrm{exc}}$, the small quantum connection of $X_{\mathrm{res}}$ induces a residual flat connection on the bundle $(V / W) \times \Delta_{\mathrm{exc}} \rightarrow \Delta_{\mathrm{exc}}$. We have a linear map $\theta: V / W \rightarrow H^{*}\left(X_{\mathrm{sm}}\right)$ which intertwines the residual flat connection with the small quantum connection of $X_{\mathrm{sm}}$ under the identification $q_{i}=\bar{q}_{i}$ of Novikov variables. More precisely, $\theta$ intertwines the action of $\left.\left(\phi_{j} \star\right)\right|_{\Delta_{\mathrm{exc}}}$ on $V / W$ with the action of $\left.\left(\bar{\phi}_{j} \star\right)\right|_{\bar{q}_{1}=q_{1}, \ldots, \bar{q}_{l}=q_{l}}$ on $H^{*}\left(X_{\mathrm{sm}}\right)$ for $1 \leq j \leq l$. Moreover $\theta$ preserves the Poincaré pairing.

(4) Let $\pi: X_{\mathrm{res}} \rightarrow X_{\mathrm{sing}}$ denote the resolution and let $r: X_{\mathrm{sm}} \rightarrow X_{\mathrm{sing}}$ denote the retraction. We have $\operatorname{Im} \pi^{*} \subset V$ and the following commutative diagram:

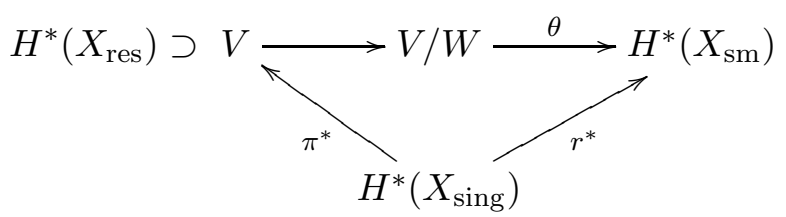

Remark 7.2. This conjecture is closely related to [1, Conjecture 4.1.2].

Appendix A. Computing quantum cohomology of A toric Variety

We explain how to compute the small quantum cohomology of a weak-Fano toric manifold using Givental's mirror theorem [7]. 
Let $X_{\text {res }}$ be the toric variety in $\$ 6$, which is a crepant resolution of a toric degeneration of $\operatorname{Gr}(2,5)$. The $I$-function of $X_{\text {res }}$ is a cohomology-valued hypergeometric function given by:

$$
I(q, z)=e^{m \log q / z} \sum_{\beta \in H_{2}\left(X_{\mathrm{res}}, \mathbb{Z}\right)} q^{\beta} \prod_{i=1}^{9} \frac{\prod_{c=-\infty}^{0}\left(R_{i}+c z\right)}{\prod_{c=-\infty}^{R_{i} \cdot \beta}\left(R_{i}+c z\right)}
$$

where we set $m \log q:=\sum_{i=1}^{3} m_{i} \log q_{i}$. In the case at hand, the mirror map is trivial and the mirror theorem of Givental [7] says that $I(q, z)$ equals the $J$-function:

$$
J(q, z)=e^{m \log q / z}\left(1+\sum_{i=0}^{N} \sum_{\beta \neq 0}\left\langle\frac{\phi_{i}}{z(z-\psi)}\right\rangle_{0,1, \beta} \phi^{i} q^{\beta}\right)
$$

where $\left\{\phi_{i}\right\}_{i=0}^{N},\left\{\phi^{i}\right\}_{i=0}^{N}$ are mutually dual bases of the cohomology as in $\oint 2$. The class $\psi$ is the first Chern class of the universal cotangent line bundle over $\bar{M}_{0,1}\left(X_{\text {res }}, \beta\right)$. More generally, the $I$-function and the $J$-function match under a change of co-ordinates (mirror map).

The method to determine the quantum product is as follows: we first find differential operators $\mathcal{D}_{i}\left(z \partial_{1}, z \partial_{2}, z \partial_{3}, z, q_{1}, q_{2}, q_{3}\right)$ which are polynomials in $z \partial_{i}:=z q_{i} \frac{\partial}{\partial q_{i}}$ and $z$ such that we have the asymptotics:

$$
\mathcal{D}_{i} I(q, z)=e^{m \log q / z}\left(\phi_{i}+O\left(z^{-1}\right)\right) \quad 0 \leq i \leq N=19 .
$$

Then the quantum product by $m_{j}, j=1,2,3$ is determined by the asymptotics:

$$
z \partial_{j}\left(\mathcal{D}_{i} I(q, z)\right)=e^{m \log q / z}\left(m_{j} \star \phi_{i}+O\left(z^{-1}\right)\right) .
$$

In our case, for the choice of a basis in (12), we can take $\mathcal{D}_{i}$ as follows:

$$
\begin{aligned}
\mathcal{D}_{0}= & 1, \mathcal{D}_{1}=z \partial_{1}, \mathcal{D}_{2}=z \partial_{2}, \mathcal{D}_{3}=z \partial_{3}, \mathcal{D}_{4}=\left(z \partial_{1}\right)^{2}, \mathcal{D}_{5}=z \partial_{1} z \partial_{2}, \\
\mathcal{D}_{6}= & z \partial_{1} z \partial_{3}, \mathcal{D}_{7}=z \partial_{2} z \partial_{3}, \mathcal{D}_{8}=\left(z \partial_{1}\right)^{3} \mathcal{D}_{9}=\left(z \partial_{1}\right)^{2} z \partial_{2}, \mathcal{D}_{10}=\left(z \partial_{1}\right)^{2} z \partial_{3}, \\
\mathcal{D}_{11}= & z \partial_{1} z \partial_{2} z \partial_{3}, \mathcal{D}_{12}=\left(z \partial_{1}\right)^{4}, \mathcal{D}_{13}=\left(z \partial_{1}\right)^{3} z \partial_{2}, \mathcal{D}_{14}=\left(z \partial_{1}\right)^{3} z \partial_{3}, \\
\mathcal{D}_{15}= & \left(z \partial_{1}\right)^{2} z \partial_{2} z \partial_{3}, \mathcal{D}_{16}=\left(z \partial_{1}\right)^{5}-q_{1}\left(1+q_{2}+q_{3}\right), \\
\mathcal{D}_{17}= & \left(z \partial_{1}\right)^{4} z \partial_{2}-q_{1} q_{2}, \mathcal{D}_{18}=\left(z \partial_{1}\right)^{4} z \partial_{3}-q_{1} q_{3}, \\
\mathcal{D}_{19}= & \left(z \partial_{1}\right)^{6}-z q_{1}\left(1+q_{2}+q_{3}\right)-q_{1}\left(1+3 q_{2}+3 q_{3}+q_{2} q_{3}\right) z \partial_{1} \\
& -q_{1}\left(2+q_{3}\right)\left(1-q_{2}\right) z \partial_{2}-q_{1}\left(2+q_{2}\right)\left(1-q_{3}\right) z \partial_{3} .
\end{aligned}
$$

\section{REFERENCES}

[1] Victor V. Batyrev, Ionuţ Ciocan-Fontanine, Bumsig Kim, and Duco van Straten. Mirror symmetry and toric degenerations of partial flag manifolds. Acta Math., 184(1):1-39, 2000.

[2] Aaron Bertram. Quantum Schubert calculus. Adv. Math., 128(2):289-305, 1997.

[3] Eduardo Cattani, Fouad El Zein, Phillip A. Griffiths, and Tráng Lê Dũng. Hodge Theory. Princeton University Press, 2014.

[4] Ionut Ciocan-Fontanine. On quantum cohomology rings of partial flag varieties. Duke Math. J., 98(3):485-524, 1999.

[5] David A. Cox and Sheldon Katz. Mirror symmetry and algebraic geometry, volume 68 of Mathematical Surveys and Monographs. American Mathematical Society, Providence, RI, 1999.

[6] Robert Friedman. Simultaneous resolution of threefold double points. Math. Ann., 274(4):671-689, 1986. 
[7] Alexander Givental. A mirror theorem for toric complete intersections. In Topological field theory, primitive forms and related topics (Kyoto, 1996), volume 160 of Progr. Math., pages 141-175. Birkhäuser Boston, Boston, MA, 1998.

[8] Alexander Givental and Bumsig Kim. Quantum cohomology of flag manifolds and Toda lattices. Comm. Math. Phys., 168(3):609-641, 1995.

[9] N. Gonciulea and V. Lakshmibai. Degenerations of flag and Schubert varieties to toric varieties. Transform. Groups, 1(3):215-248, 1996.

[10] Yujiro Kawamata. Unobstructed deformations. A remark on a paper of Z. Ran: "Deformations of manifolds with torsion or negative canonical bundle" [J. Algebraic Geom. 1 (1992), no. 2, 279-291; MR1144440 (93e:14015)]. J. Algebraic Geom., 1(2):183-190, 1992.

[11] Yuan-Pin Lee, Hui-Wen Lin, and Chin-Lung Wang. $A+B$ theory in conifold tranition for CalabiYau threefolds. arXiv:1502.03277 [math.AG], 2015.

[12] An-Min Li and Yongbin Ruan. Symplectic surgery and Gromov-Witten invariants of Calabi-Yau 3-folds. Invent. Math., 145(1):151-218, 2001.

[13] Yu. I. Manin. Generating functions in algebraic geometry and sums over trees. In The moduli space of curves (Texel Island, 1994), volume 129 of Progr. Math., pages 401-417. Birkhäuser Boston, Boston, MA, 1995.

[14] David R. Morrison. Through the looking glass. In Mirror symmetry, III (Montreal, PQ, 1995), volume 10 of AMS/IP Stud. Adv. Math., pages 263-277. Amer. Math. Soc., Providence, RI, 1999.

[15] Yoshinori Namikawa. Smoothing Fano 3-folds. J. Algebraic Geom., 6(2):307-324, 1997.

[16] I. Smith, R. P. Thomas, and S.-T. Yau. Symplectic conifold transitions. J. Differential Geom., 62(2):209-242, 2002.

[17] Gang Tian. Smoothing 3-folds with trivial canonical bundle and ordinary double points. In Essays on mirror manifolds, pages 458-479. Int. Press, Hong Kong, 1992.

E-mail address: iritani@math.kyoto-u.ac.jp

E-mail address: xiao@math.kyoto-u.ac.jp 